\title{
A VERSATILE ANNULATION PROTOCOL TOWARDS NOVEL CONSTRAINED PHOSPHINIC PEPTIDOMIMETICS
}

\author{
Magdalini Nasopoulou, ${ }^{\dagger}$ Dimitris Georgiadis, ${ }^{\dagger}$ Magdalini Matziari, ${ }^{\dagger}$ Vincent Dive ${ }^{\ddagger}$ and \\ Athanasios Yiotakis ${ }^{\dagger, *}$ \\ ${ }^{\dagger}$ Department of Chemistry, Laboratory of Organic Chemistry, \\ University of Athens, Panepistimiopolis Zografou, \\ 15771, Athens, Greece and ${ }^{\ddagger}$ CEA, Département d'Ingénierie et d'Etudes des Protéines, 91191 \\ Gif/Yvette Cedex, France \\ yiotakis@chem.uoa.gr
}

\section{SUPPORTING INFORMATION}

TABLE OF CONTENTS

\begin{tabular}{ll}
\hline \multicolumn{1}{c}{ PART I } & \\
\hline Table of Contents & $\mathrm{S}-1$ \\
General Procedures & $\mathrm{S}-3$ \\
Experimental Data & $\mathrm{S}-4$ \\
${ }^{1} \mathrm{H}$ and ${ }^{13} \mathrm{C}$ NMR spectra of compound $\mathbf{S 1}$ & $\mathrm{S}-11$ \\
${ }^{1} \mathrm{H}$ and ${ }^{13} \mathrm{C}$ NMR spectra of compound $\mathbf{S 2}$ & $\mathrm{S}-12$ \\
${ }^{1} \mathrm{H}$ and ${ }^{13} \mathrm{C}$ NMR spectra of compound $\mathbf{S 3}$ & $\mathrm{S}-13$ \\
${ }^{1} \mathrm{H}$ and ${ }^{13} \mathrm{C}$ NMR spectra of compound $\mathbf{S 4}$ & $\mathrm{S}-14$ \\
${ }^{1} \mathrm{H}$ and ${ }^{13} \mathrm{C}$ NMR spectra of compound $\mathbf{S 5}$ & $\mathrm{S}-15$ \\
${ }^{1} \mathrm{H}$ and ${ }^{13} \mathrm{C}$ NMR spectra of compound $\mathbf{S 6}$ & $\mathrm{S}-16$ \\
${ }^{1} \mathrm{H}$ NMR spectrum of compound $\mathbf{5 a}$ & $\mathrm{S}-17$ \\
${ }^{13} \mathrm{C}$ NMR spectrum of compound $\mathbf{5 a}$ & $\mathrm{S}-18$ \\
${ }^{31} \mathrm{P}$ NMR spectrum and HPLC profile of compound $\mathbf{5 a}$ & $\mathrm{S}-19$ \\
${ }^{1} \mathrm{H}$ NMR spectrum of compound $\mathbf{5 b}$ & $\mathrm{S}-20$ \\
${ }^{13} \mathrm{C}$ NMR spectrum of compound $\mathbf{5 b}$ & $\mathrm{S}-21$ \\
${ }^{31} \mathrm{P}$ NMR spectrum and HPLC profile of compound $\mathbf{5 b}$ & $\mathrm{S}-22$ \\
${ }^{1} \mathrm{H}$ NMR spectrum of compound $\mathbf{5 c}$ & $\mathrm{S}-23$ \\
${ }^{13} \mathrm{C}$ NMR spectrum of compound $\mathbf{5 c}$ & $\mathrm{S}-24$ \\
${ }^{31} \mathrm{P}$ NMR spectrum and HPLC profile of compound $\mathbf{5 c}$ & $\mathrm{S}-25$ \\
${ }^{1} \mathrm{H}$ NMR spectrum of compound $\mathbf{5 e}$ & $\mathrm{S}-26$
\end{tabular}


${ }^{13} \mathrm{C}$ NMR spectrum of compound $\mathbf{5 e}$

${ }^{31} \mathrm{P}$ NMR spectrum and HPLC profile of compound $\mathbf{5 e}$

S-28

\section{PART II}

\begin{tabular}{lc}
\hline${ }^{1} \mathrm{H}$ NMR spectrum of compound $\mathbf{5 f}$ & $\mathrm{S}-29$ \\
${ }^{13} \mathrm{C}$ NMR spectrum of compound $\mathbf{5 f}$ & $\mathrm{S}-30$ \\
${ }^{31} \mathrm{P}$ NMR spectrum and HPLC profile of compound $\mathbf{5 f}$ & $\mathrm{S}-31$ \\
${ }^{1} \mathrm{H}$ and ${ }^{13} \mathrm{C}$ NMR spectra of compound $\mathbf{6}$ & $\mathrm{S}-32$ \\
${ }^{1} \mathrm{H}$ and ${ }^{13} \mathrm{C}$ NMR spectra of compound $\mathbf{7}$ & $\mathrm{S}-33$ \\
${ }^{1} \mathrm{H}$ and ${ }^{13} \mathrm{C}$ NMR spectra of compound $\mathbf{8}$ & $\mathrm{S}-34$ \\
${ }^{1} \mathrm{H}$ and ${ }^{13} \mathrm{C}$ NMR spectra of compound $\mathbf{9}$ & $\mathrm{S}-35$ \\
${ }^{1} \mathrm{H}$ and ${ }^{13} \mathrm{C}$ NMR and HPLC profile of compound $\mathbf{1 0}$ & $\mathrm{S}-36$ \\
${ }^{1} \mathrm{H}$ NMR spectrum of compound $\mathbf{5 d}$ & $\mathrm{S}-37$ \\
${ }^{13} \mathrm{C}$ NMR spectrum of compound $\mathbf{5 d}$ & $\mathrm{S}-38$ \\
${ }^{31} \mathrm{P}$ NMR spectrum and HPLC profile of compound $\mathbf{5 d}$ & $\mathrm{S}-39$ \\
${ }^{1} \mathrm{H}$ NMR spectrum of compound $\mathbf{1 7} \mathbf{a}$ & $\mathrm{S}-40$ \\
${ }^{13} \mathrm{C}$ NMR spectrum of compound $\mathbf{1 7} \mathbf{a}$ & $\mathrm{S}-41$ \\
${ }^{31} \mathrm{P}$ NMR spectrum and HPLC profile of compound $\mathbf{1 7} \mathbf{a}$ & $\mathrm{S}-42$ \\
\hline
\end{tabular}

\section{PART III}

\begin{tabular}{|c|c|}
\hline${ }^{1} \mathrm{H}$ NMR spectrum of compound $\mathbf{1 7 c}$ & S-43 \\
\hline${ }^{13} \mathrm{C}$ NMR spectrum of compound $\mathbf{1 7 c}$ & S-44 \\
\hline${ }^{31} \mathrm{P}$ NMR spectrum and HPLC profile of compound $\mathbf{1 7} \mathbf{c}$ & S-45 \\
\hline${ }^{1} \mathrm{H}$ NMR spectrum of compound $\mathbf{1 8 a}$ & S-46 \\
\hline${ }^{13} \mathrm{C}$ NMR spectrum of compound $\mathbf{1 8 a}$ & S-47 \\
\hline${ }^{1} \mathrm{H}$ NMR spectrum of compound $\mathbf{1 8 c}$ & S-48 \\
\hline${ }^{13} \mathrm{C}$ NMR spectrum of compound $\mathbf{1 8 c}$ & S-49 \\
\hline${ }^{31} \mathrm{P}$ NMR spectra of compounds $\mathbf{1 8 a}$ and $\mathbf{1 8 c}$ & S-50 \\
\hline MS spectra of compounds $\mathbf{1 8 a}$ and $\mathbf{1 8 c}$ & S-51 \\
\hline
\end{tabular}

\section{PART IV}

${ }^{1} \mathrm{H}$ and ${ }^{31} \mathrm{P}$ NMR spectra and HPLC profile of compound 21

S-52

${ }^{13} \mathrm{C}$ NMR spectrum of compound 21

${ }^{1} \mathrm{H}$ NMR spectrum of compound $\mathbf{2 3}$

${ }^{13} \mathrm{C}$ NMR spectrum of compound $\mathbf{2 3}$

S-55

${ }^{1} \mathrm{H}$ and ${ }^{31} \mathrm{C}$ NMR spectra of compound 24

S-56

${ }^{31} \mathrm{P}$ NMR spectrum and HPLC profile of compound 24

S-57

${ }^{1} \mathrm{H}$ NMR spectrum of compound 19

S-58

${ }^{13} \mathrm{C}$ NMR spectrum of compound 19 
${ }^{31} \mathrm{P}$ NMR and MS spectra of compound 19

S-60

${ }^{1} \mathrm{H}$ NMR spectrum of compound 25

S-61

${ }^{13} \mathrm{C}$ NMR spectrum of compound 25

S-62

${ }^{31} \mathrm{P}$ NMR and MS spectra of compound 25

S-63

General Procedures. Dry tetrahydrofuran (THF), and methylene chloride $\left(\mathrm{CH}_{2} \mathrm{Cl}_{2}\right)$ were obtained by distillation of commercially available predried solvents from $\mathrm{NaH}$. All of the compounds, for which analytical and spectroscopic data are quoted, were chromatographically homogenous. Reagents were purchased at the highest commercial quality and used without further purification. Reactions were monitored by thin-layer chromatography (TLC) carried out on $0.25 \mathrm{~mm}$ silica gel plates (60F-254) using UV light as visualizing agent and an aqueous solution of cerium molybdate/ $\mathrm{H}_{2} \mathrm{SO}_{4}$ ("Blue Stain"), and heat as developing agent. Purification of compounds by column chromatography was carried out on silica gel (70-230 mesh). RPHPLC analyses were carried out on an analytical instrument using a MZ-Analytical Column 250x4mm, Kromasil $100(\mathrm{C} 18,5 \mu \mathrm{m})$, at a flow rate of $0.5 \mathrm{~mL} / \mathrm{min}$. Solvent $\mathrm{A}: 10 \% \mathrm{CH}_{3} \mathrm{CN}_{\text {, }}$ 90\% $\mathrm{H}_{2} \mathrm{O}, 0.1 \%$ TFA. Solvent B: $90 \% \mathrm{CH}_{3} \mathrm{CN}, 10 \% \mathrm{H}_{2} \mathrm{O}, 0.09 \%$ TFA. The following gradients were used: 1. $t=0 \min (0 \% \mathrm{~B}), t=10 \min (25 \% \mathrm{~B}), t=45 \min (75 \% \mathrm{~B}), t=50 \min (100 \% \mathrm{~B}), t=55$ $\min (100 \%$ B), $t=60 \min (40 \%$ B) for compounds $\mathbf{5 a}, \mathbf{5 b}, \mathbf{5 c}, \mathbf{5 d}, \mathbf{5 e}$ and $\mathbf{2 4} ; 2 . t=0 \mathrm{~min}(45 \% \mathrm{~B})$, $t=40 \min (75 \% \mathrm{~B}), t=45 \min (100 \% \mathrm{~B}), t=50 \min (100 \% \mathrm{~B}), t=60 \min (40 \% \mathrm{~B})$ for compound 17a; $3 . t=0 \min (50 \% \mathrm{~B}), t=40 \min (80 \% \mathrm{~B}), t=45 \min (100 \% \mathrm{~B}), t=50 \min (100 \% \mathrm{~B}), t=60 \min$ (40\% B) for compounds $\mathbf{5 f}$ and $\mathbf{1 7 c}$; Eluted peaks were detected by a UV detector at $254 \mathrm{~nm}$. Reported retention times (where more than one) correspond to different diastereoisomers and are counted in minutes. In NMR measurements, $\mathrm{CDCl}_{3}, \mathrm{~d}_{6}-\mathrm{DMSO}$ and $\mathrm{CD}_{3} \mathrm{OD}$ were used as solvents. ${ }^{1} \mathrm{H},{ }^{31} \mathrm{P}$ and ${ }^{13} \mathrm{C}$ NMR spectra were recorded on a $200 \mathrm{MHz}$ spectrometer. Proton and carbon chemical shifts are referenced to residual solvent. ${ }^{31} \mathrm{P}$ chemical shifts are reported on $\delta$ scale (in ppm) downfield from $85 \% \mathrm{H}_{3} \mathrm{PO}_{4}$. The following abbreviations were used to explain 
the NMR multiplicities: $\mathrm{s}=$ singlet, $\mathrm{d}=$ doublet, $\mathrm{t}=$ triplet, $\mathrm{q}=$ quartet, $\mathrm{m}=$ multiplet, $\mathrm{br}=$ broad. All spectroscopy and analytical data were obtained in the Laboratory of Organic Chemistry of the University of Athens, except HRMS spectra for compounds 8, 18a, 18c, 19, 23 and 25 that were measured in the LMP Laboratory of the University of Montpellier II, Montpellier, France.

\section{Experimental Data.}

General method for the synthesis of Fmoc-protected amino alcohols S1-S6

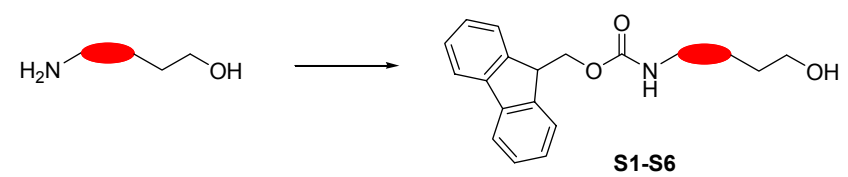

In a mixture of the appropriate amino alcohol $(1.00 \mathrm{mmol})$ and $\mathrm{NaHCO}_{3} 10 \%(3 \mathrm{~mL})$ in dioxane $(1.5 \mathrm{~mL})$, a solution of Fmoc-Cl $(388 \mathrm{mg}, 1.50 \mathrm{mmol})$ in dioxane $(1.5 \mathrm{~mL})$ is slowly added at $0^{\circ} \mathrm{C}$. The mixture is vigorously stirred at rt overnight. Then, AcOEt $(30 \mathrm{~mL})$ and $\mathrm{HCl} 1 \mathrm{M}(15$ $\mathrm{mL}$ ) are added, the organic layer is separated, washed with brine and dried over $\mathrm{Na}_{2} \mathrm{SO}_{4}$. Evaporation of the solvent and purification by column chromatography, using $\mathrm{CHCl}_{3}: \mathrm{MeOH}=$ 98:2 as eluent, affords the pure products (S1-S6) as white solids. Yields given below. (In the case of S6, 2.50mmol of Fmoc-Cl were used).

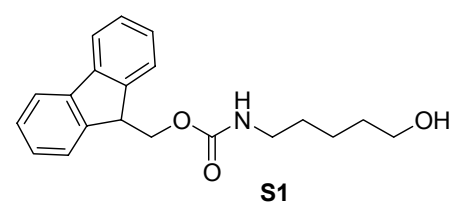

9H-Fluoren-9-ylmethyl $N$-(5-hydroxypentyl)carbamate: Yield $82 \%$; white solid; TLC $R_{\mathrm{f}}=0.23\left(\mathrm{CHCl}_{3}: \mathrm{MeOH}=95: 5\right) ;{ }^{1} \mathrm{H}$ NMR $\left(200 \mathrm{MHz}, \mathrm{CDCl}_{3}\right) \delta 1.30-$ $1.58(\mathrm{~m}, 6 \mathrm{H}), 2.83(\mathrm{br} \mathrm{s}, 1 \mathrm{H}), 3.05-3.22(\mathrm{~m}, 2 \mathrm{H}), 3.57(\mathrm{t}, J=6.6 \mathrm{~Hz}, 2 \mathrm{H}), 4.18(\mathrm{br} \mathrm{t}, J=6.6 \mathrm{~Hz}$, 1H), 4.38 (br d, $J=6.6 \mathrm{~Hz}, 2 \mathrm{H}), 5.16-5.32(\mathrm{~m}, 1 \mathrm{H}), 7.24-7.75(\mathrm{~m}, 8 \mathrm{H}) ;{ }^{13} \mathrm{C} \mathrm{NMR}(50 \mathrm{MHz}$, 
$\left.\mathrm{CDCl}_{3}\right) \delta 22.7,29.4,31.9,40.7,47.0,62.0,66.2,119.7,124.8,126.8,127.4,141.0,143.7,156.5 ;$ ESMS $m / z$ calcd for $\mathrm{C}_{20} \mathrm{H}_{23} \mathrm{NO}_{3} \mathrm{Na}(\mathrm{M}+\mathrm{Na})^{+} 348.2$, found 348.1.

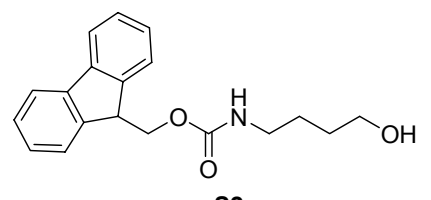

9H-Fluoren-9-ylmethyl $N$-(4-hydroxybutyl)carbamate: Yield 90\%; white solid; TLC $R_{\mathrm{f}}=0.21\left(\mathrm{CHCl}_{3}: \mathrm{MeOH}=95: 5\right) ;{ }^{1} \mathrm{H}$ NMR $\left(200 \mathrm{MHz}, \mathrm{CDCl}_{3}\right) \delta 1.48-1.69(\mathrm{~m}$, 4H), $3.15-3.37(\mathrm{~m}, 2 \mathrm{H}), 3.61-3.78(\mathrm{~m}, 2 \mathrm{H}), 4.23(\mathrm{br} \mathrm{t}, J=6.6 \mathrm{~Hz}, 1 \mathrm{H}), 4.43(\mathrm{br} \mathrm{d}, J=6.6 \mathrm{~Hz}$, 2H), 7.26 - $7.80(\mathrm{~m}, 8 \mathrm{H}) ;{ }^{13} \mathrm{C} \mathrm{NMR}\left(50 \mathrm{MHz}, \mathrm{CDCl}_{3}\right) \delta 26.0,29.2,40.5,46.9,49.9,61.6,66.2$, 119.7, 124.7, 126.8, 127.4, 141.0, 143.7, 156.7; ESMS $m / z$ calcd for $\mathrm{C}_{19} \mathrm{H}_{22} \mathrm{NO}_{3}(\mathrm{M}+\mathrm{H})^{+} 312.2$, found 312.2 .

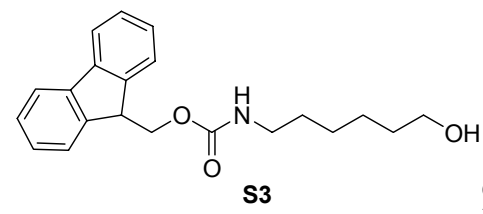

9H-Fluoren-9-ylmethyl $\quad N$-(6-hydroxyhexyl)carbamate: Yield 95\%; white solid; TLC $R_{\mathrm{f}}=0.35\left(\mathrm{CHCl}_{3}: \mathrm{MeOH}=95: 5\right) ;{ }^{1} \mathrm{H}$ NMR $\left(200 \mathrm{MHz}, \mathrm{CDCl}_{3}\right) \delta 1.27-$ $1.59(\mathrm{~m}, 8 \mathrm{H}), 3.11-3.24(\mathrm{~m}, 2 \mathrm{H}), 3.64(\mathrm{t}, J=6.6 \mathrm{~Hz}, 2 \mathrm{H}), 4.23($ br t, $J=7.3 \mathrm{~Hz}, 1 \mathrm{H}), 4.42$ (br d, $J=7.3 \mathrm{~Hz}, 2 \mathrm{H}), 4.79(\mathrm{br}, 1 \mathrm{H}), 7.27-7.79(\mathrm{~m}, 8 \mathrm{H}) ;{ }^{13} \mathrm{C} \mathrm{NMR}\left(50 \mathrm{MHz}, \mathrm{CDCl}_{3}\right) \delta 25.3,26.3$, 29.7, 29.9, 35.5, 40.9, 47.3, 62.7, 66.5, 119.9, 125.0, 127.0, 127.6, 141.3, 144.0, 156.5; ESMS $m / z$ calcd for $\mathrm{C}_{21} \mathrm{H}_{26} \mathrm{NO}_{3}(\mathrm{M}+\mathrm{H})^{+}$340.2, found 340.1.

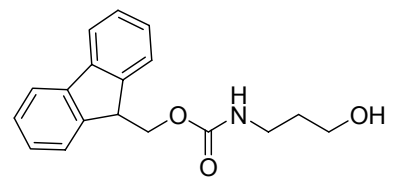

S4

9H-Fluoren-9-ylmethyl $N$-(3-hydroxypropyl)carbamate: Yield 92\%; white solid; TLC $R_{\mathrm{f}}=0.20\left(\mathrm{CHCl}_{3}: \mathrm{MeOH}=95: 5\right) ;{ }^{1} \mathrm{H}$ NMR $\left(200 \mathrm{MHz}, \mathrm{CDCl}_{3}\right) \delta 1.57-1.74(\mathrm{~m}$, 2H), 2.95 (br s, 1H), $3.20-3.38(\mathrm{~m}, 2 \mathrm{H}), 3.61(\mathrm{t}, J=5.9 \mathrm{~Hz}, 2 \mathrm{H}), 4.18($ br t, $J=6.6 \mathrm{~Hz}, 1 \mathrm{H})$, $4.41(\mathrm{brd}, J=6.6 \mathrm{~Hz}, 2 \mathrm{H}), 5.22-5.39(\mathrm{~m}, 1 \mathrm{H}), 7.25-7.76(\mathrm{~m}, 8 \mathrm{H}) ;{ }^{13} \mathrm{C} \mathrm{NMR}\left(50 \mathrm{MHz}, \mathrm{CDCl}_{3}\right) \delta$ $32.3,37.6,47.1,59.3,66.5,119.9,124.9,126.9,127.6,141.2,143.8,157.2$; ESMS $m / z$ calcd for $\mathrm{C}_{18} \mathrm{H}_{19} \mathrm{NO}_{3} \mathrm{Na}(\mathrm{M}+\mathrm{Na})^{+}$320.1, found 320.0. 


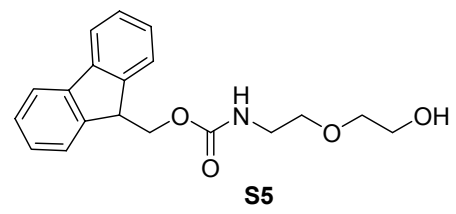

9H-Fluoren-9-ylmethyl $N$-[2-(2-hydroxyethoxy)ethyl]carbamate: Yield 96\%; white solid; TLC $R_{\mathrm{f}}=0.22\left(\mathrm{CHCl}_{3}: \mathrm{MeOH}=95: 5\right) ;{ }^{1} \mathrm{H}$ NMR $\left(200 \mathrm{MHz}, \mathrm{CDCl}_{3}\right) \delta$ $3.18-3.37(\mathrm{~m}, 2 \mathrm{H}), 3.42-3.52(\mathrm{~m}, 4 \mathrm{H}), 3.58-3.67(\mathrm{~m}, 2 \mathrm{H}), 4.18($ br t $, J=6.6 \mathrm{~Hz}, 1 \mathrm{H}), 4.42(\mathrm{br}$ $\mathrm{d}, J=6.6 \mathrm{~Hz}, 2 \mathrm{H}), 5.75-5.96(\mathrm{~m}, 1 \mathrm{H}), 7.25-7.76(\mathrm{~m}, 8 \mathrm{H}) ;{ }^{13} \mathrm{C} \mathrm{NMR}\left(50 \mathrm{MHz}, \mathrm{CDCl}_{3}\right) \delta 40.6$, 47.0, 61.3, 66.1, 69.9, 72.1, 119.8, 124.8, 126.9, 127.5, 141.1, 143.7, 156.6; ESMS $\mathrm{m} / \mathrm{z}$ calcd for $\mathrm{C}_{19} \mathrm{H}_{22} \mathrm{NO}_{4}(\mathrm{M}+\mathrm{H})^{+}$350.2, found 350.3.

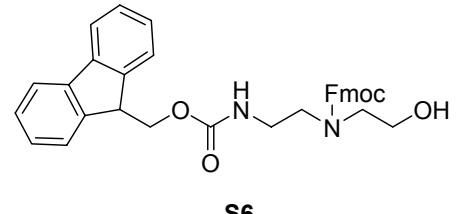

S6

ylmethoxy)carbonyl]amino\}ethyl)- $N$-(2-hydroxyethyl)-carbamate. Yield $85 \%$; white solid; TLC $R_{\mathrm{f}}=0.36\left(\mathrm{CHCl}_{3}: \mathrm{MeOH}=95: 5\right) ;{ }^{1} \mathrm{H}$ NMR $\left(200 \mathrm{MHz}, \mathrm{CDCl}_{3}\right) \delta 2.79-2.98(\mathrm{~m}, 2 \mathrm{H}), 3.12-$ $3.51(\mathrm{~m}, 5 \mathrm{H}), 3.55-3.67(\mathrm{~m}, 1 \mathrm{H}), 4.12-4.56(\mathrm{~m}, 6 \mathrm{H}), 7.22-7.82(\mathrm{~m}, 16 \mathrm{H}) ;{ }^{13} \mathrm{C} \mathrm{NMR}(50 \mathrm{MHz}$, $\left.\mathrm{CDCl}_{3}\right) \delta 39.2,39.5,46.8,47.9,48.3,50.7,51.1,60.4,65.8,66.3,66.9,119.6,124.3,124.5$, $124.8,126.7,127.3,140.9,143.5,156.3,156.6,156.7$; ESMS $m / z$ calcd for $\mathrm{C}_{34} \mathrm{H}_{33} \mathrm{~N}_{2} \mathrm{O}_{5} \mathrm{Na}$ $(\mathrm{M}+\mathrm{Na})^{+}$571.2, found 571.3.

\section{2-Benzyl-3-[\{1-[(9H-fluoren-9-ylmethoxy)carbonyl]-2-}

piperidinyl\}(hydroxy)phosphoryl]propanoic acid (5a). ${ }^{13} \mathrm{C} \mathrm{NMR}\left(50 \mathrm{MHz}, \mathrm{CDCl}_{3}\right) \delta 20.4$, $22.9,25.0,27.1,28.1,39.5,41.0,41.6,47.1,48.6,49.3,50.9,51.5,67.9,120.0,124.9,126.6$, $127.1,127.7,128.4,128.9,137.5,141.2,143.7,155.6,178.9$.

\section{2-Benzyl-3-[\{1-[(9H-fluoren-9-ylmethoxy)carbonyl]-2-}

pyrrolidinyl\}(hydroxy)phosphoryl]propanoic acid (5b). ${ }^{13} \mathrm{C} \mathrm{NMR}\left(50 \mathrm{MHz}, \mathrm{CDCl}_{3}\right) \delta 24.5$, $25.2,28.3,29.6,30.3,39.3,39.6,41.1,47.0,54.8,55.5,56.9,57.6,67.8,119.9,125.0,126.5$, $127.0,127.7,128.3,129.0,137.8,141.2,143.6,143.7,155.8,178.3$. 
2-Benzyl-3-[\{1-[(9H-fluoren-9-ylmethoxy)carbonyl]-2-

azepanyl\}(hydroxy)phosphoryl]propanoic acid (5c). ${ }^{13} \mathrm{C} N \mathrm{NR}\left(50 \mathrm{MHz}, \mathrm{d}_{6}\right.$-DMSO) $\delta 24.6$, $24.8,25.6,26.1,27.5,28.1,29.0,43.0,43.3,46.9,52.4,52.9,54.5,55.0,66.5,120.1,124.8$, $124.9,125.4,126.3,127.1,127.6,128.2,129.0,129.0,138.8,138.9,140.8,140.9,143.6,143.8$, $144.1,144.2,154.7,156.0,175.1,175.3$.

2-Benzyl-3-[(5S)-5-benzyl-1-[(9H-fluoren-9-ylmethoxy)carbonyl]tetrahydro-1H-pyrrol-3yl(hydroxy)phosphoryl]propanoic acid (5d). ${ }^{13} \mathrm{C} \mathrm{NMR}\left(50 \mathrm{MHz}, \mathrm{CDCl}_{3}\right) \delta 23.3,28.0,29.3$, $31.0,31.9,37.8,38.5,39.5,40.6,41.0,41.5,47.3,56.0,56.6,57.9,58.5,59.6,66.8,120.1,124.5$, $124.6,125.0,126.0,126.3,126.6,127.2,127.7,128.3,128.4,129.1,129.4,137.4,137.8,137.9$, $138.0,138.4,141.3,143.5,143.6,155.7,155.9,178.3$

2-Benzyl-3-[\{4-[(9H-fluoren-9-ylmethoxy)carbonyl]-1,4-oxazinan-3-

yl\}(hydroxy)phosphoryl]propanoic acid (5e): ${ }^{13} \mathrm{C} \mathrm{NMR}\left(50 \mathrm{MHz}, \mathrm{CDCl}_{3}\right) \delta 29.3,29.6,30.2$, $30.9,39.5,40.8,41.2,47.0,49.1,49.9,51.1,51.9,52.6,53.4,64.1,66.1,67.9,120.0,124.8$, $126.5,127.1,127.7,128.3,128.9,129.0,137.6,137.6,141.2,143.4,155.2,177.6$.

\section{2-Benzyl-3-[\{1,4-bis [(9H-fluoren-9-ylmethoxy)carbonyl]-2-}

piperazinyl $\}$ (hydroxy)phosphoryl]propanoic acid (5f). ${ }^{13} \mathrm{C} \mathrm{NMR}\left(50 \mathrm{MHz}, \mathrm{CDCl}_{3}\right) \delta 28.5$, $29.3,30.1,30.3,31.4,31.9,39.4,40.7,41.3,43.3,47.0,47.1,48.2,49.2,50.4,51.4,68.0,119.9$, $120.0,124.8,124.9,125.1,126.6,127.1,127.6,127.8,128.4,129.0,137.5,141.1,141.2,141.3$, $143.5,144.0,155.2,177.7$

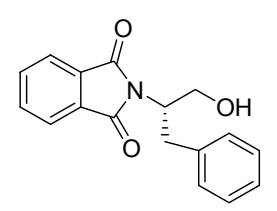

6

2-[(1S)-1-Benzyl-2-hydroxyethyl]-1H-isoindole-1,3(2H)-dione. A mixture of phthalic anhydride (800 mg, $5.40 \mathrm{mmol})$ and 2-amino-3-phenyl-propan-1-ol [Gage, J.; Evans, D. Organic Syntheses, 1993, Coll. Vol. 8,528$](817 \mathrm{mg}, 5.40 \mathrm{mmol})$ is heated to $135-140^{\circ} \mathrm{C}$ in an open flask for $30 \mathrm{~min}$. A stream of argon is applied to expel water vapor. After cooling to room 
temperature the reaction residue is dissolved in AcOEt $(100 \mathrm{~mL})$, washed with $\mathrm{NaHCO}_{3} 5 \%(3 \mathrm{x}$ $40 \mathrm{~mL}), \mathrm{HCl} 1 \mathrm{M}(3 \times 40 \mathrm{~mL}), \mathrm{H}_{2} \mathrm{O}(2 \times 40 \mathrm{~mL})$ and brine and dried over $\mathrm{Na}_{2} \mathrm{SO}_{4}$. The solvent is evaporated and the crude product is purified by column chromatography with $\mathrm{Et}_{2} \mathrm{O}$ to afford compound $6(1.41 \mathrm{~g}, 93 \%)$ as a white solid; TLC $R_{\mathrm{f}}=0.50($ chloroform $/$ methanol $=95: 5) ;{ }^{1} \mathrm{H}$ $\operatorname{NMR}\left(200 \mathrm{MHz}, \mathrm{CDCl}_{3}\right) \delta 3.04(\mathrm{~s}, 1 \mathrm{H}), 3.17(\mathrm{~d}, J=9.0 \mathrm{~Hz}, 2 \mathrm{H}), 3.90(\mathrm{dd}, J=4.1,11.9 \mathrm{~Hz}, 1 \mathrm{H})$, $4.08(\mathrm{dd}, J=7.8,11.9 \mathrm{~Hz}, 1 \mathrm{H}), 4.50-4.71(\mathrm{~m}, 1 \mathrm{H}), 7.11-7.19(\mathrm{~m}, 5 \mathrm{H}), 7.62-7.75(\mathrm{~m}, 4 \mathrm{H}) ;{ }^{13} \mathrm{C}$ NMR $\left(50 \mathrm{MHz}, \mathrm{CDCl}_{3}\right) \delta 34.6,55.1,62.5,123.2,126.5,128.4,128.9,131.4,133.9,137.3,168.9$; ESMS $m / z$ calcd for $\mathrm{C}_{17} \mathrm{H}_{16} \mathrm{NO}_{3}(\mathrm{M}+\mathrm{H})^{+} 282.1$, found 282.0 .

(2S)-2-(1,3-dioxo-1,3-dihydro-2H-isoindol-2-yl)-3-phenylpropanal (7). ${ }^{13} \mathrm{C} \mathrm{NMR}$ (50MHz, $\left.\mathrm{CDCl}_{3}\right) \delta 32.9,59.8,123.5,126.9,128.3,128.5,128.8,131.3,133.9,134.2,135.9,167.5,196.1$.

Ethyl (4R)-4-(1,3-dioxo-1,3-dihydro-2H-isoindol-2-yl)-5-phenylpentanoate $(8) .{ }^{13} \mathrm{C}$ NMR $\left(50 \mathrm{MHz}, \mathrm{CDCl}_{3}\right) \delta 14.0,27.1,31.4,38.5,52.6,60.5,123.0,126.5,128.4,128.8,131.4,133.8$, $137.7,168.3,172.5$

(4R)-4-Amino-5-phenylpentanoic acid (9). ${ }^{13} \mathrm{C} \mathrm{NMR}\left(50 \mathrm{MHz}, \mathrm{CD}_{3} \mathrm{OD}\right) \delta 27.6,30.6,38.9,52.8$, $127.3,128.9,129.2,130.9,131.2,135.9,176.0$.

9H-Fluoren-9-ylmethyl $\quad N$-[(1R)-1-benzyl-4-hydroxybutyl]carbamate $\quad(10) . \quad{ }^{13} \mathrm{C} \quad \mathrm{NMR}$ $\left(50 \mathrm{MHz}, \mathrm{CDCl}_{3}\right) \delta 28.9,30.6,41.3,47.3,51.8,62.4,66.2,119.9,125.0,126.4,127.0,127.6$, $128.4,129.4,137.7,141.3,143.9,156.0$.

2-Benzyl-3-oxo-3-(2-oxo-2-phenylethoxy)propyl\{1-[(9H-fluoren-9-ylmethoxy)carbonyl]-2piperidinyl\}phosphinic acid (17a). ${ }^{13} \mathrm{C} \mathrm{NMR}\left(50 \mathrm{MHz}, \mathrm{CDCl}_{3}\right) \delta 20.5,22.6,22.9,25.1,27.8$, $29.3,38.9,40.3,40.4,41.7,47.2,48.9,49.4,50.0,51.0,51.5,52.0,66.1,67.9,119.9,125.0$, $126.6,127.1,127.7,127.8,128.4,128.7,129.1,133.8,134.0,137.8,137.8,141.2,143.8,143.9$, $154.9,155.6,173.3,173.4,192.3$.

2-Benzyl-3-oxo-3-(2-oxo-2-phenylethoxy)propyl\{1-[(9H-fluoren-9-ylmethoxy)carbonyl]-2azepanyl\}phosphinic acid (17c). ${ }^{13} \mathrm{C} \mathrm{NMR}\left(50 \mathrm{MHz}, \mathrm{CDCl}_{3}\right) \delta$ 24.9, 25.2, 25.7, 26.1, 27.2, 
$27.9,29.0,29.2,38.8,38.9,39.3,40.5,43.8,47.2,52.8,53.2,54.9,55.3,66.1,67.1,67.6,119.9$, $124.7,124.9,126.5,127.0,127.7,127.8,128.3,128.7,129.2,129.2,133.8,134.0,137.9,141.3$, $141.3,143.6,143.7,144.0,156.9,173.3,173.4,192.2,192.3$.

3-((1-Adamantyloxy)\{1-[(9H-fluoren-9-ylmethoxy)carbonyl]-2-piperidinyl $\}$ phosphoryl)-2benzylpropanoic acid (18a). ${ }^{13} \mathrm{C} \mathrm{NMR}\left(50 \mathrm{MHz}, \mathrm{CDCl}_{3}\right) \delta$ 20.0, 20.4, 23.5, 23.8, 25.1, 28.3, $30.0,30.1,31.0,31.1,31.2,31.4,31.9,35.5,35.6,39.2,39.4,41.0,41.8,44.2,44.3,44.3,44.4$, $47.2,50.2,52.5,67.8,83.3,83.5,84.4,84.6,119.9,125.1,126.4,126.5,127.0,127.1,127.6$, 127.7, 128.3, 129.1, 129.4, 138.2, 138.4, 141.3, 143.7, 143.8, 143.9, 144.1, 155.7, 175.3, 175.4, 175.6 .

3-((1-Adamantyloxy)\{1-[(9H-fluoren-9-ylmethoxy)carbonyl]-2-azepanyl\}phosphoryl)-2benzylpropanoic acid (18c). ${ }^{13} \mathrm{C} \mathrm{NMR}\left(50 \mathrm{MHz}, \mathrm{CDCl}_{3}\right) \delta 25.0,25.9,26.6,27.1,27.7,28.0$, 28.7, 29.3, 30.2, 31.1, 31.8, 35.5, 38.7, 39.3, 39.7, 40.83, 41.2, 41.6, 43.9, 44.2, 47.4, 52.7, 52.9, $53.4,54.8,55.2,55.7,66.7,67.0,67.5,83.2,83.5,84.1,84.4,119.9,124.4,124.8,125.5,126.4$, $127.0,127.7,128.4,129.2,129.5,138.6,141.4,143.9,144.2,155.2,156.6,157.2,174.8,175.3$.

2-[(2-Oxo-2-phenylethoxy)carbonyl]-4-pentynylphosphinic acid (21). ${ }^{13} \mathrm{C} \mathrm{NMR}(50 \mathrm{MHz}$, $\left.\mathrm{CDCl}_{3}\right) \delta 22.0,28.9,30.8,37.7,66.5,71.6,79.8,80.0,127.7,128.3,128.8,133.7,134.0,134.2$, $172.0,191.7$.

Methyl (2S)-2-\{[(benzyloxy)carbonyl](tert-butoxycarbonyl)amino\}-5-oxopentanoate (23). ${ }^{13} \mathrm{C} \mathrm{NMR}\left(50 \mathrm{MHz}, \mathrm{CDCl}_{3}\right) \delta 22.4,27.8,40.4,52.3,57.7,69.0,84.1,128.4,128.5,128.6,135.0$, $151.2,153.7,170.4,200.8$

(5S)-1-[(Benzyloxy)carbonyl]-5-(methoxycarbonyl)tetrahydro-1H-pyrrol-2-yl\{2-[(2-oxo-2phenylethoxy)carbonyl]-4-pentynyl\}phosphinic acid (24). ${ }^{13} \mathrm{C} \mathrm{NMR}\left(50 \mathrm{MHz}, \mathrm{CDCl}_{3}\right) \delta 22.5$, $23.9,25.1,27.9,28.8,29.6,30.0,37.7,38.0,52.0,52.2,53.4,56.0,56.2,58.2,58.5,60.1,66.3$, $67.7,71.1,80.1,127.7,128.0,128.1,128.3,128.4,128.7,133.8,134.0,135.7,154.5,155.0$, $172.2,172.3,172.7,191.9$ 
1-Benzyl 2-methyl (2S)-5-((1-adamantyloxy)\{2-[(2-oxo-2-phenylethoxy)carbonyl]-4pentynyl $\}$ phosphoryl)tetrahydro-1 $\boldsymbol{H}$-pyrrole-1,2-dicarboxylate (19). ${ }^{13} \mathrm{C} \mathrm{NMR}(50 \mathrm{MHz}$, $\left.\mathrm{CDCl}_{3}\right) \delta 22.4,22.6,22.8,24.2,24.4,25.7,28.0,28.3,29.3,29.4,29.6,30.2,30.7,31.1,31.8$, $32.1,35.6,36.1,36.3,38.8,39.6,41.1,43.0,43.1,44.3,52.0,52.2,56.3,57.2,57.7,58.4,59.4$, $59.9,60.0,66.3,67.4,67.6,70.8,70.9,80.4,80.6,83.2,83.4,127.7,128.1,128.4,128.8,133.7$, $134.2,136.0,154.5,155.0,172.7,191.8$.

2-(\{(1-Adamantyloxy)[(5S)-1-[(benzyloxy)carbonyl]-5-(methoxycarbonyl)tetrahydro-1Hpyrrol-2-yl]phosphoryl\}methyl)-4-pentynoic acid (25). ${ }^{13} \mathrm{C} \mathrm{NMR}\left(50 \mathrm{MHz}, \mathrm{CDCl}_{3}\right) \delta 22.6$, $22.8,24.4,25.8,28.0,29.3,29.6,30.1,30.2,31.2,31.8,35.5,36.3,36.7,38.2,38.7,39.4,43.1$, $43.3,44.4,44.5,44.6,52.1,52.3,55.6,57.3,57.8,59.5,59.7,59.9,67.6,67.9,70.4,70.6,81.0$, $84.3,85.3,128.2,128.4,128.4,128.5,129.0,135.8,154.6,155.2,172.8,173.8$. 

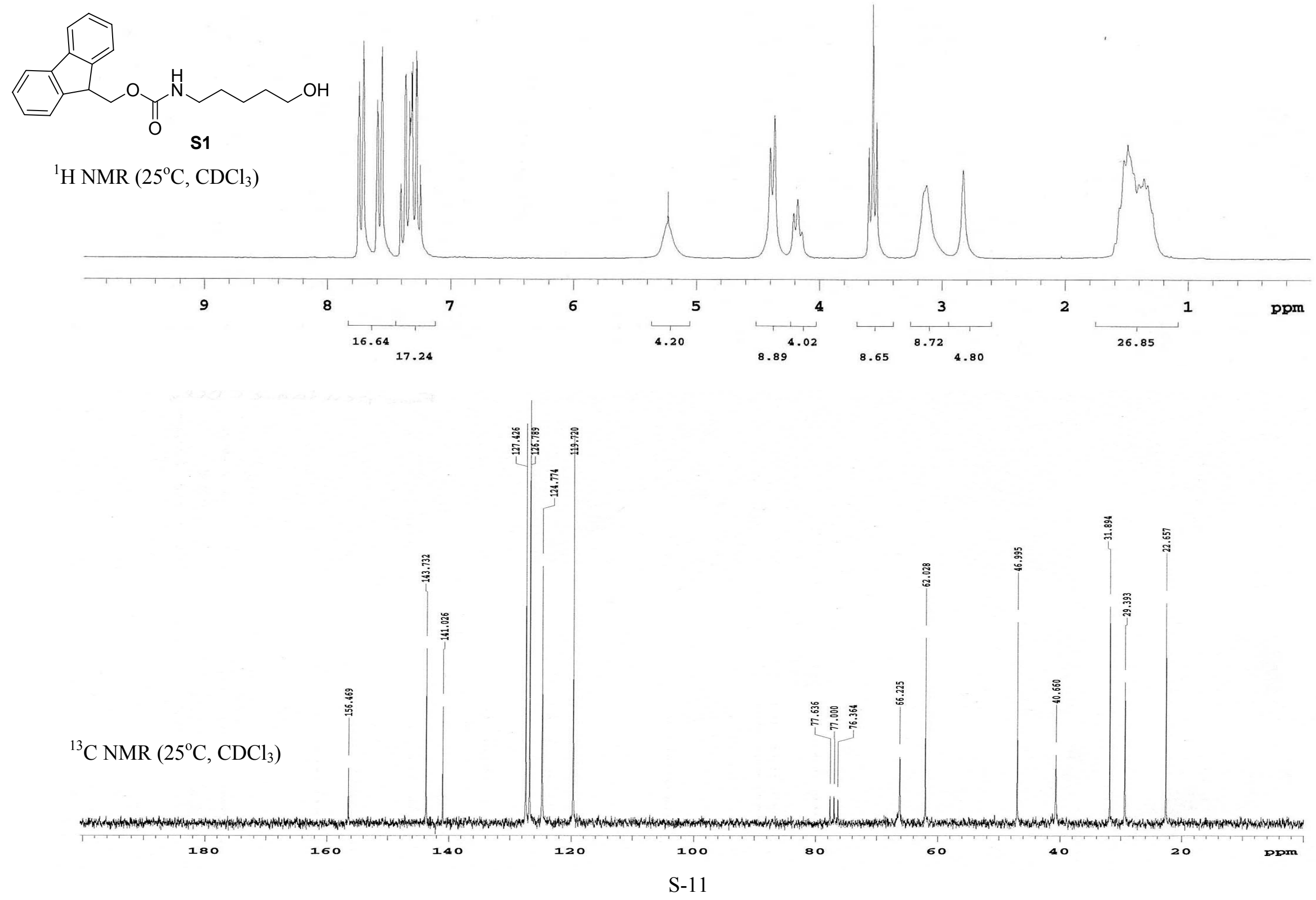

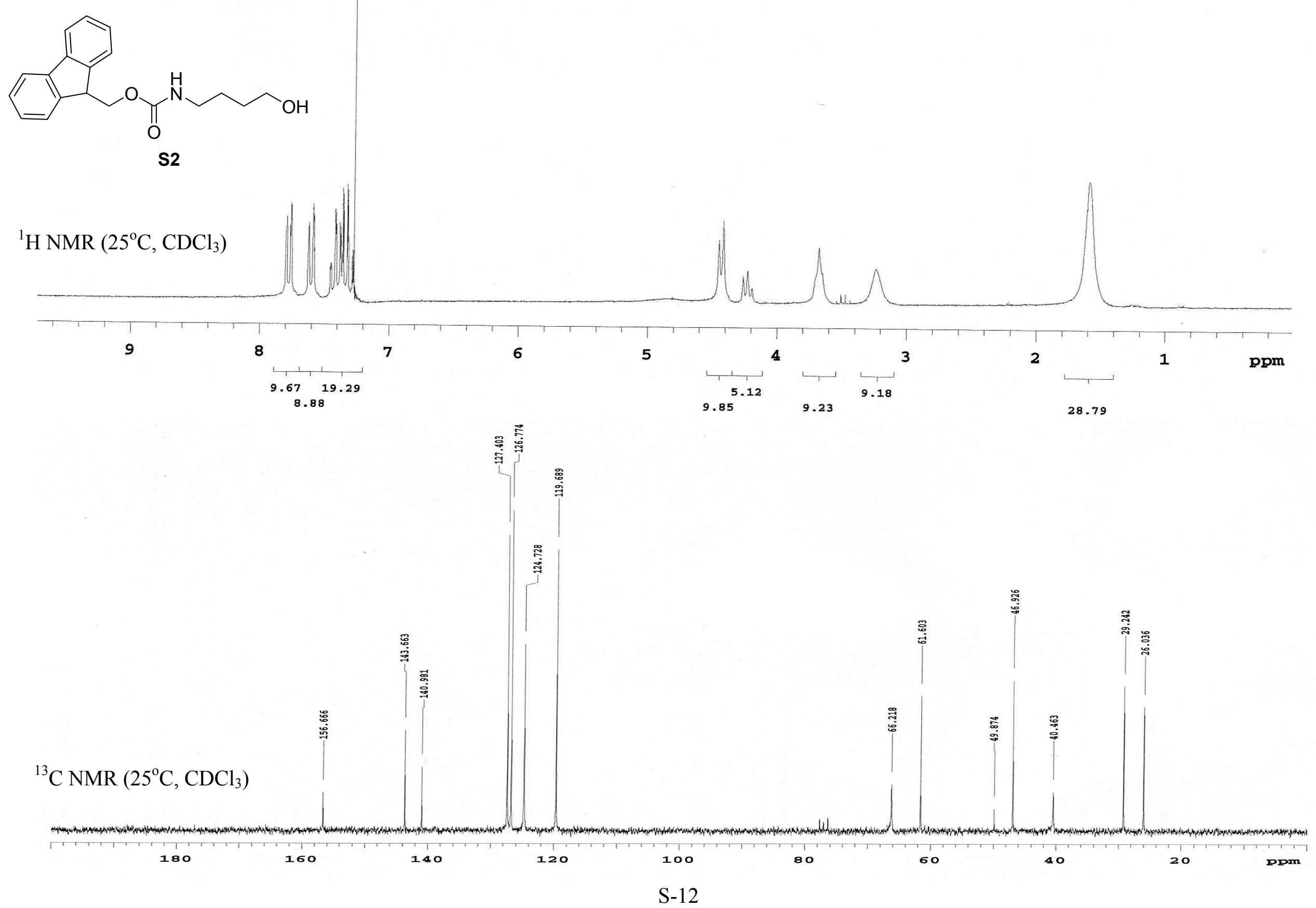

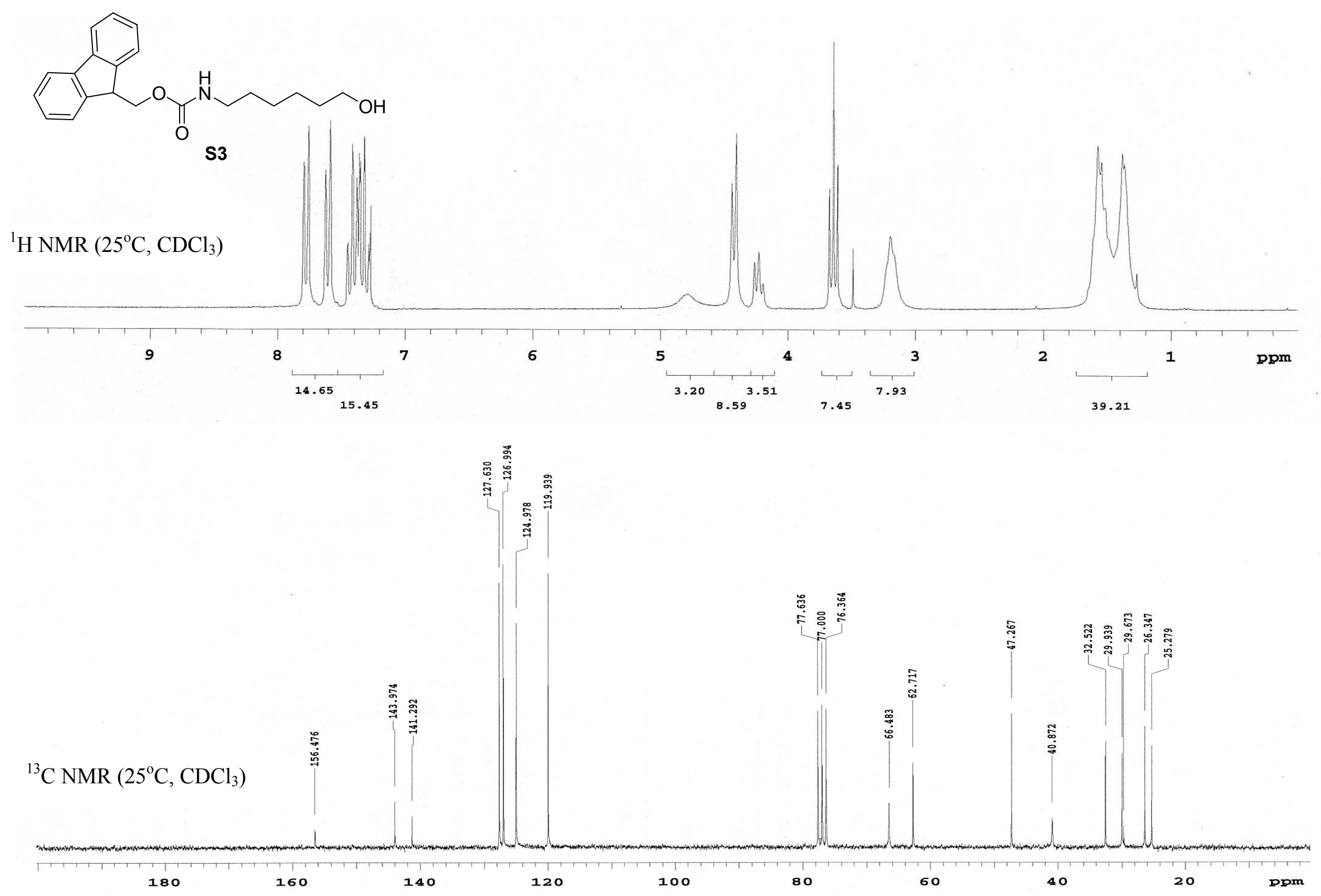


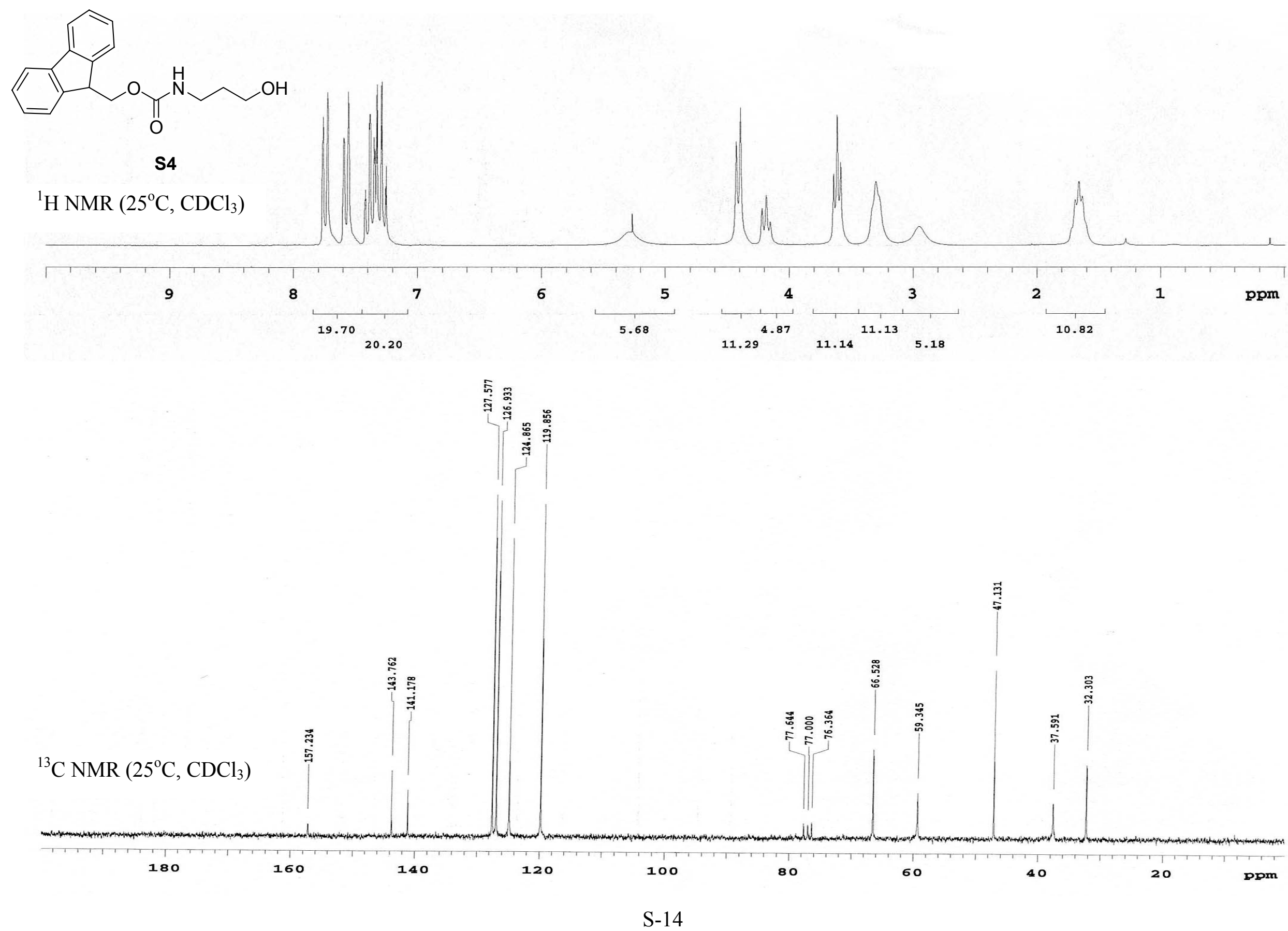




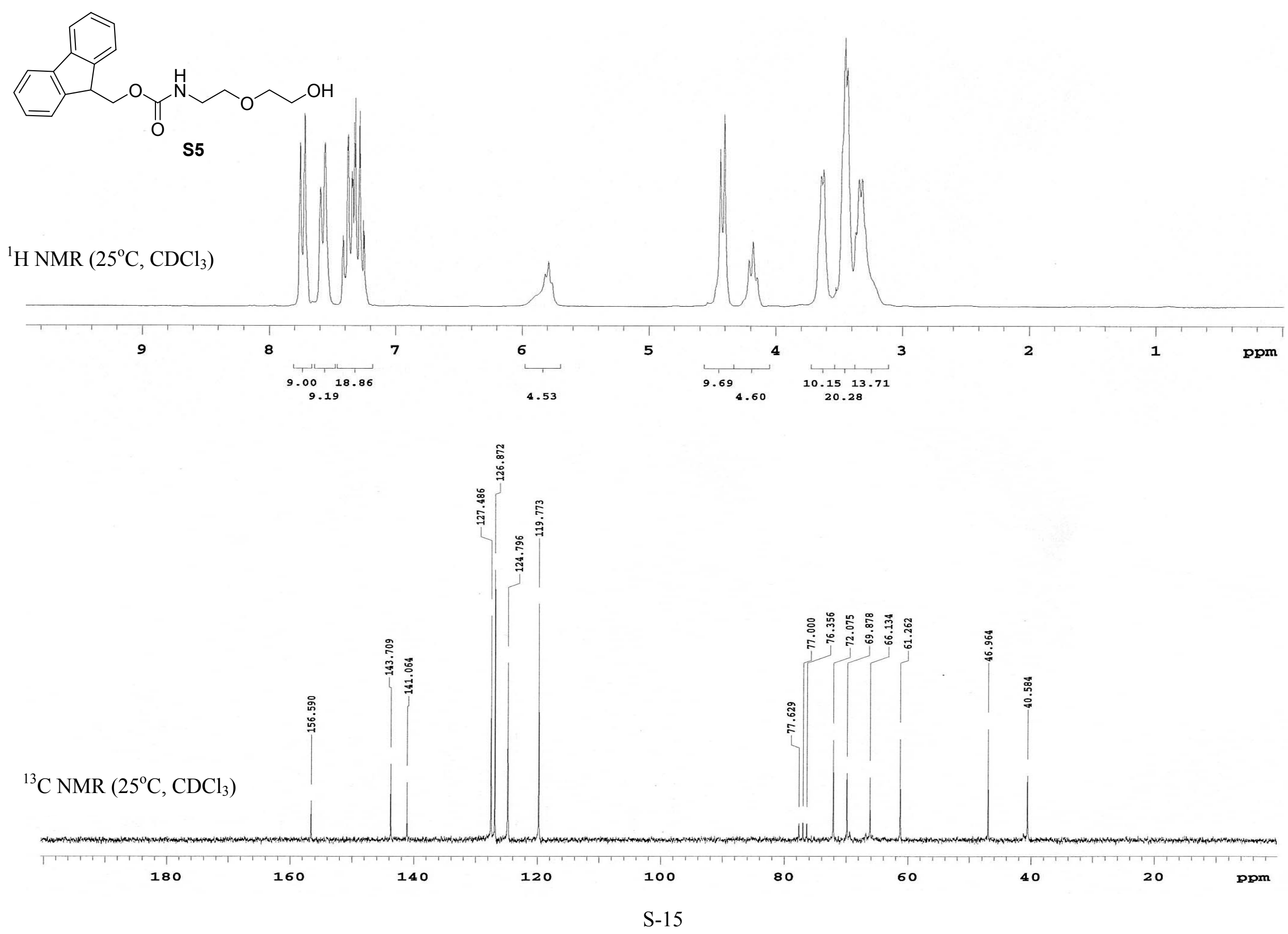




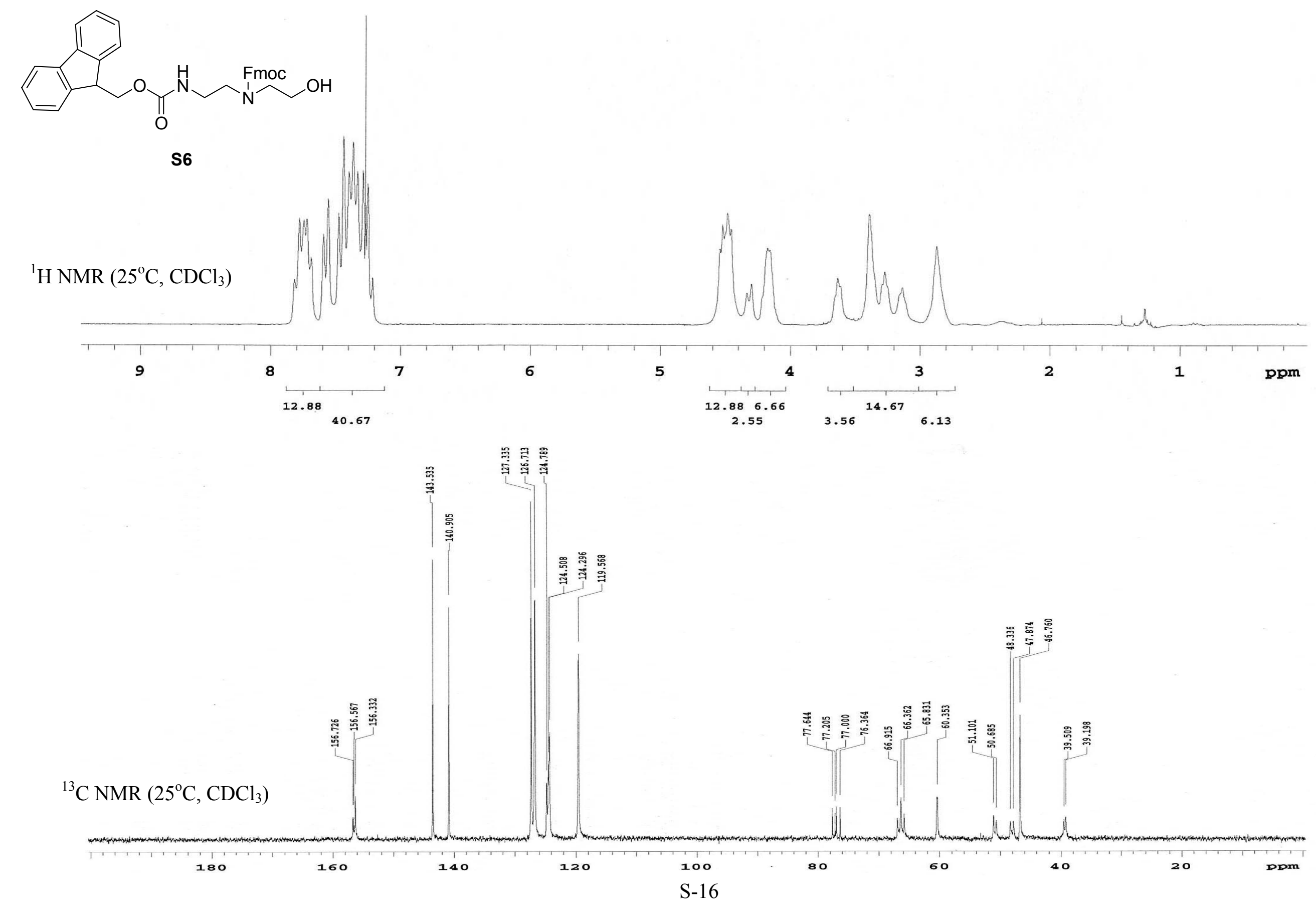




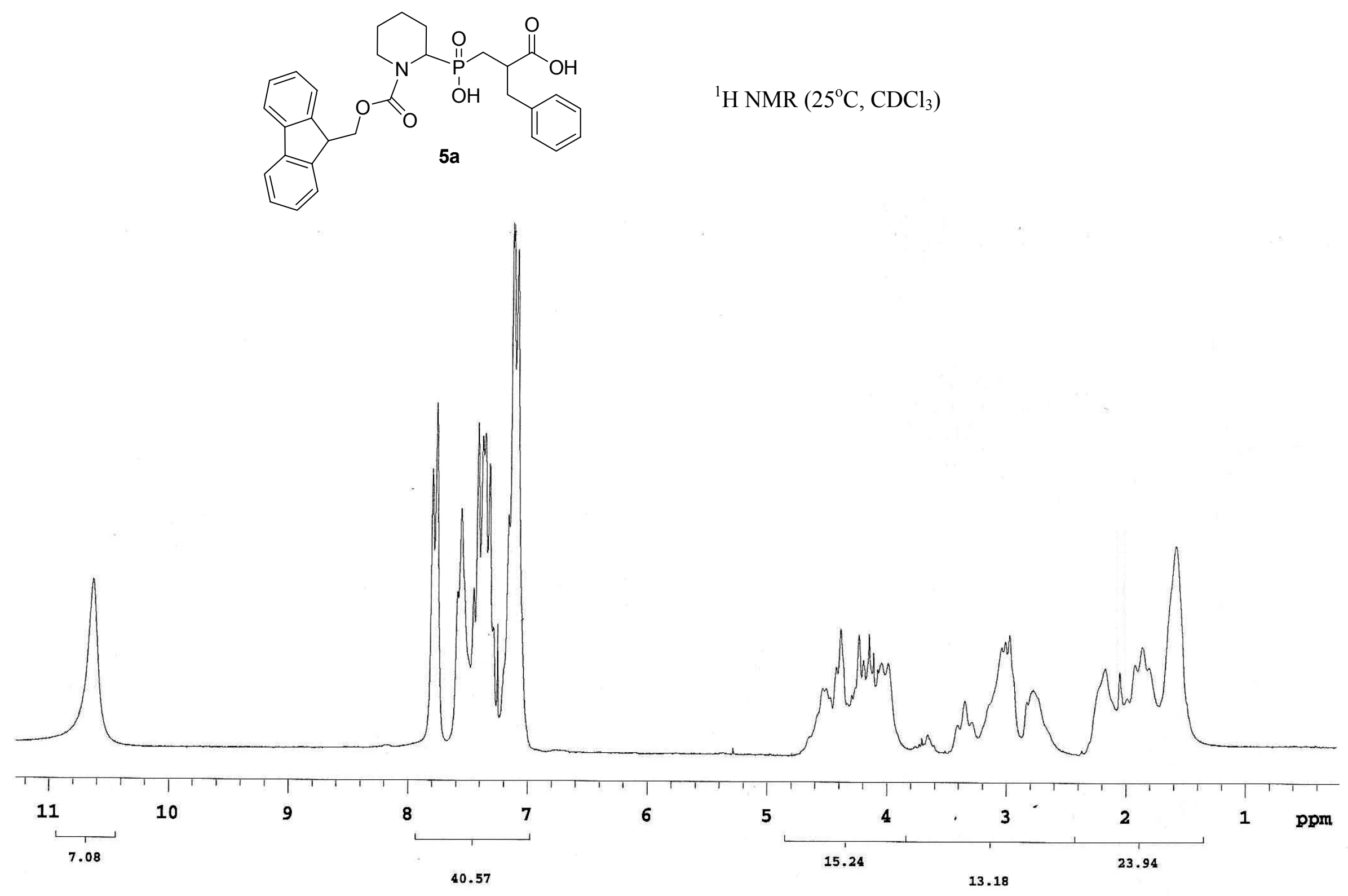




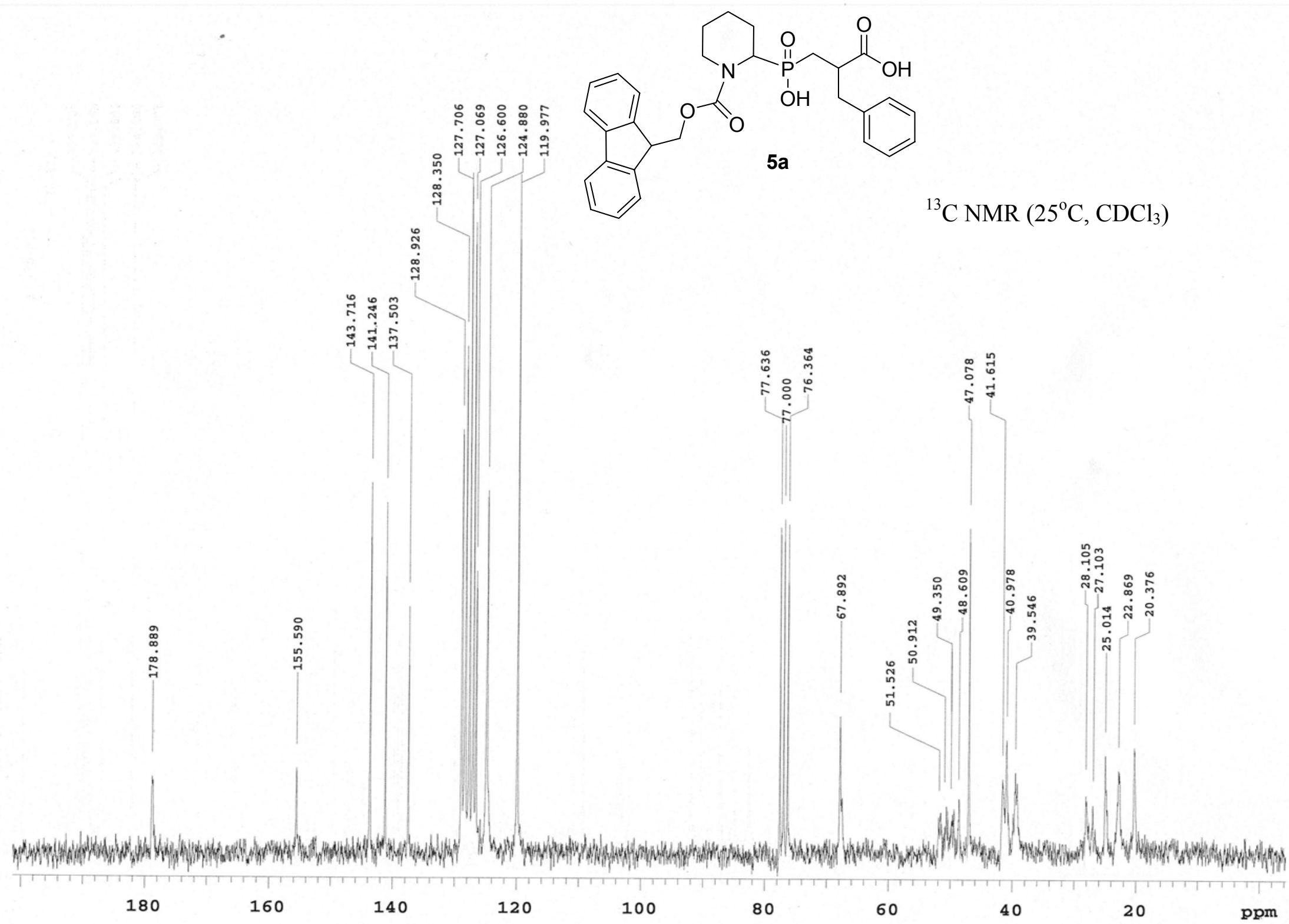



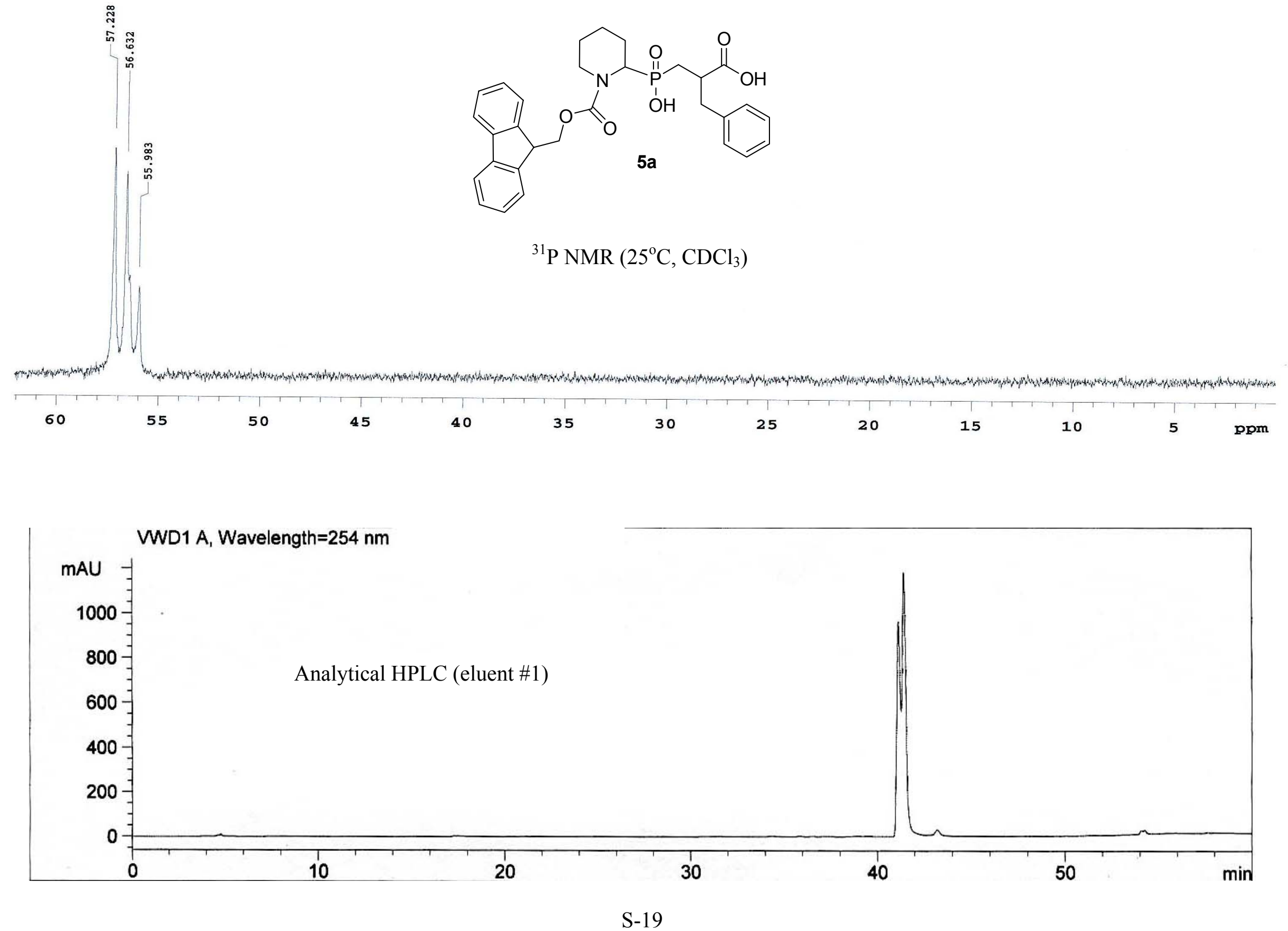


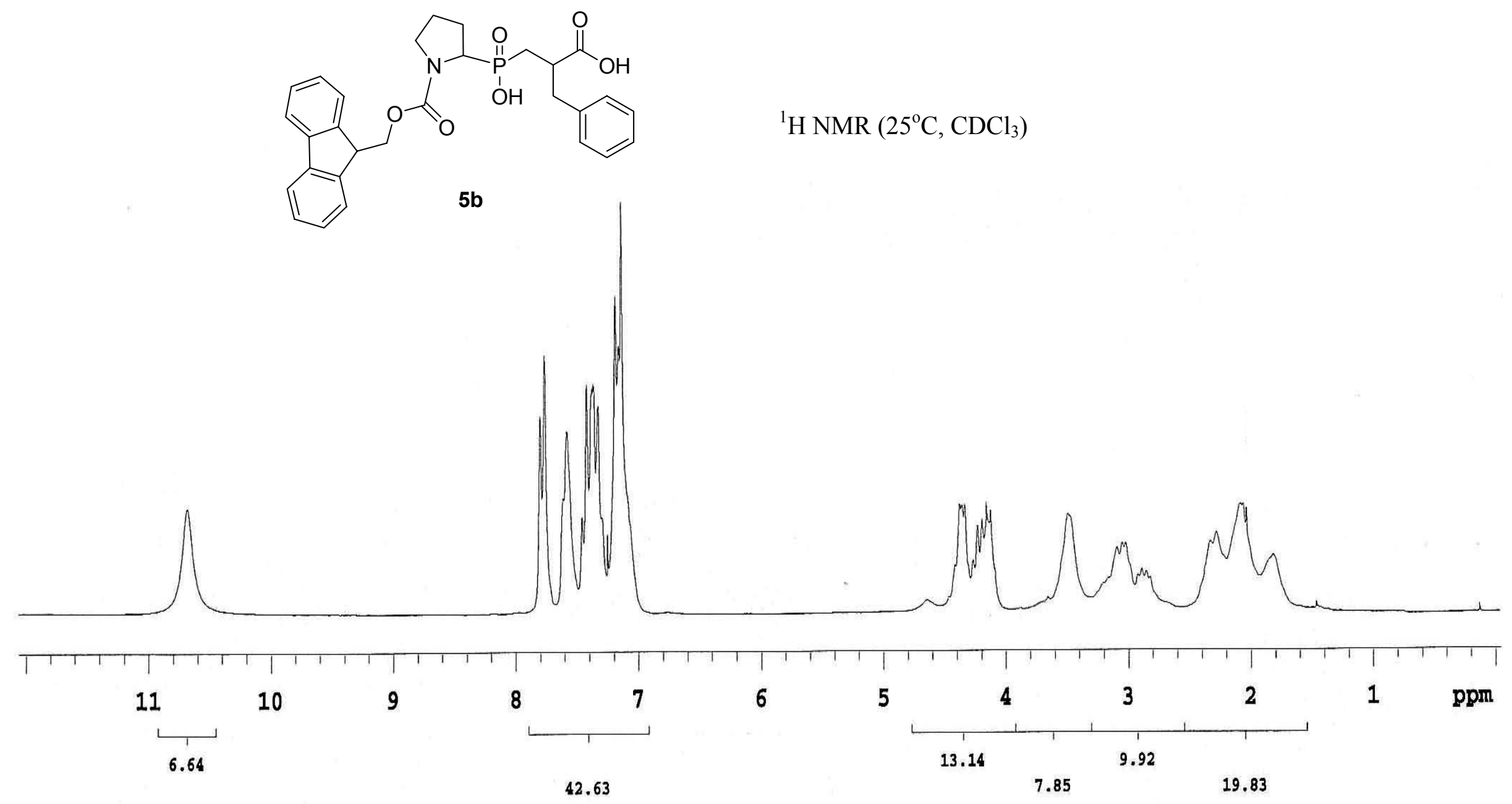




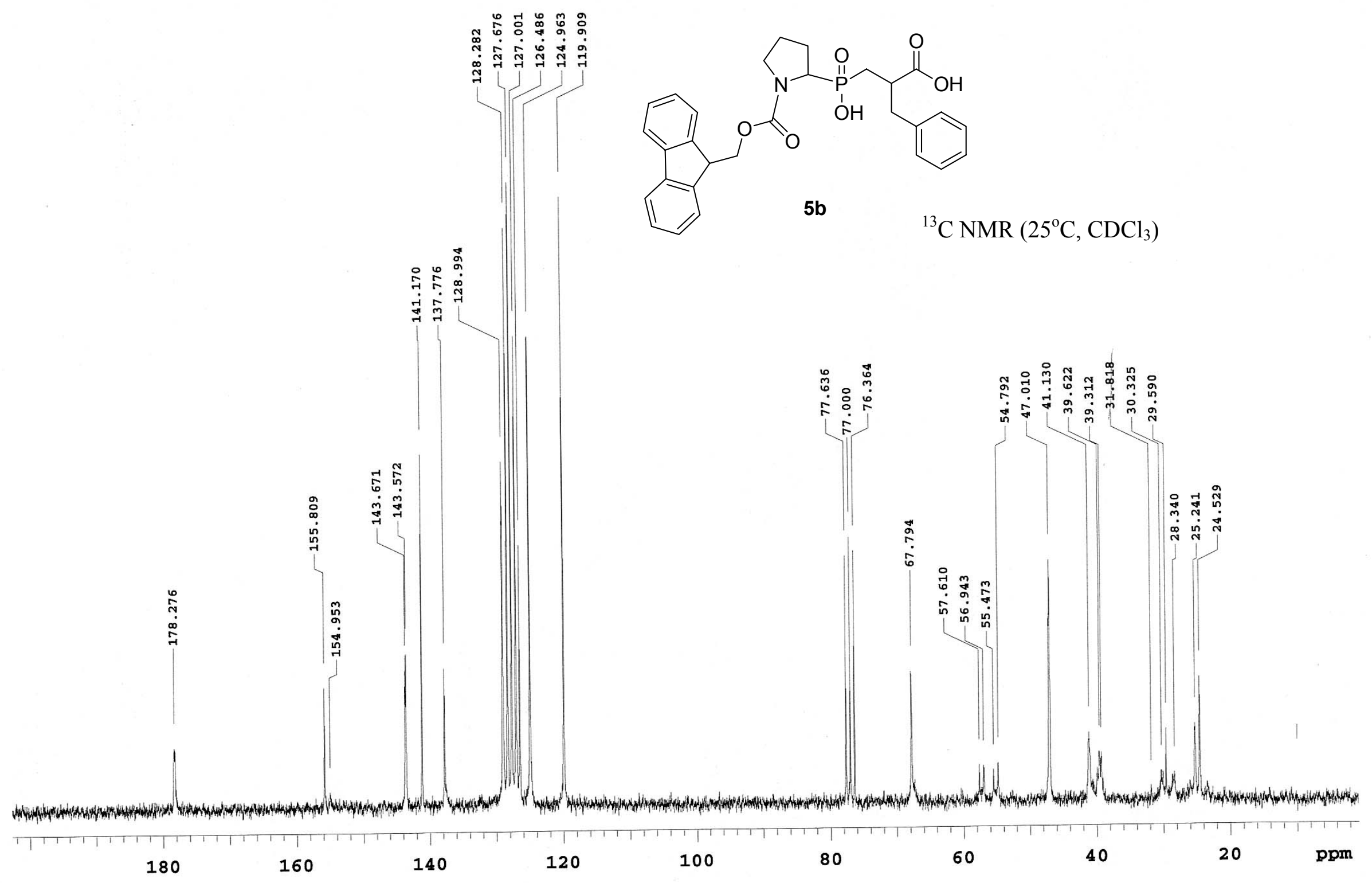



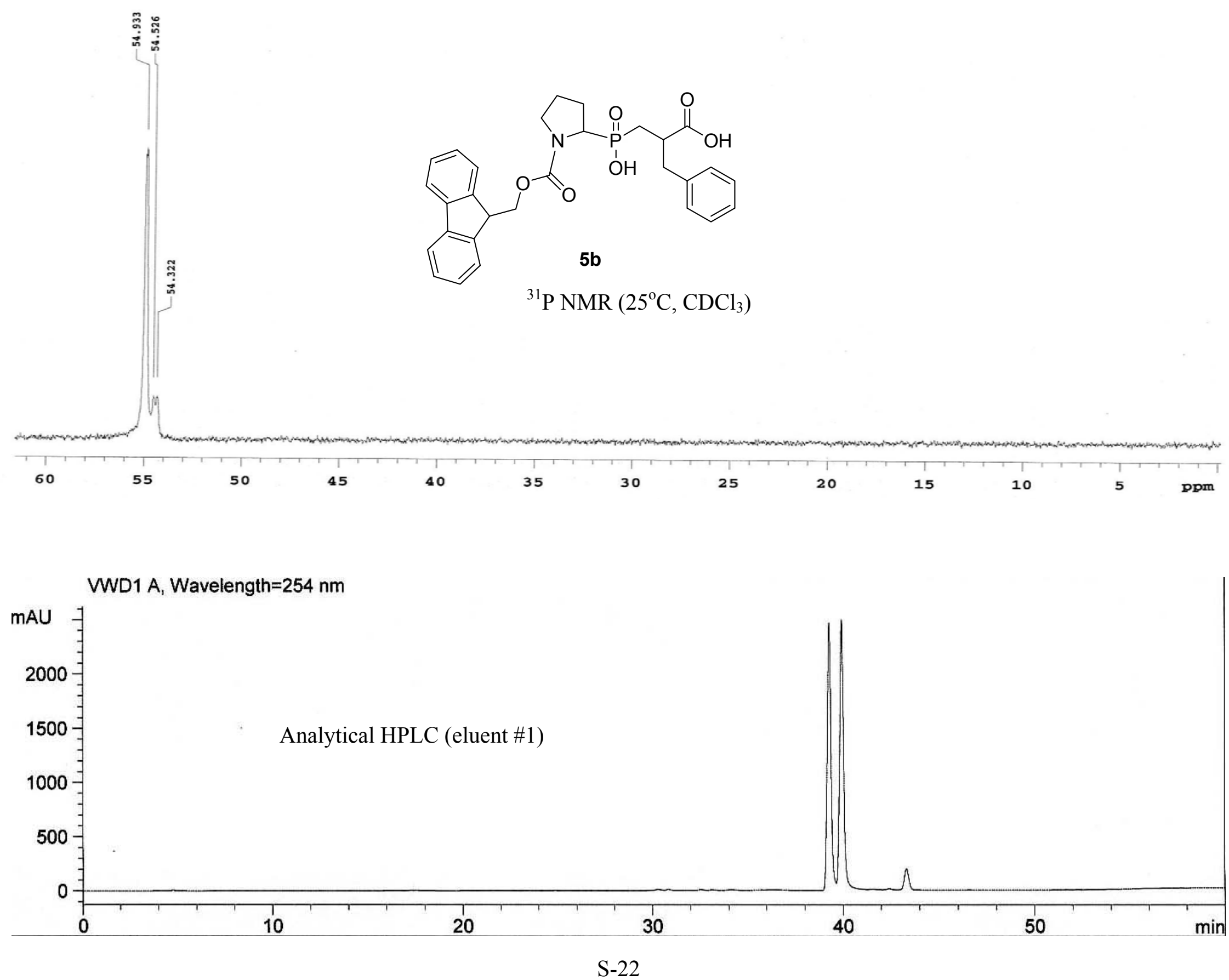


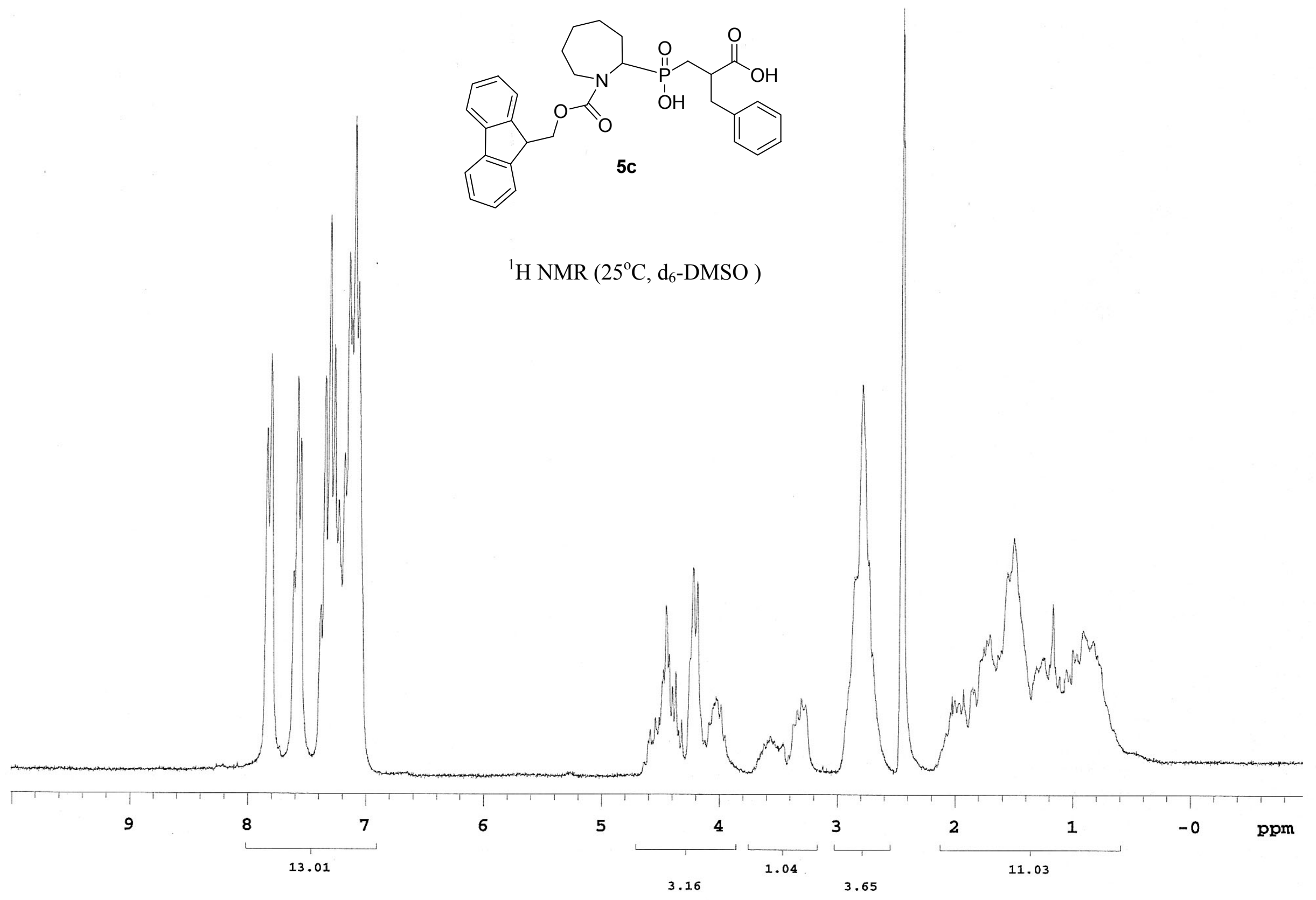




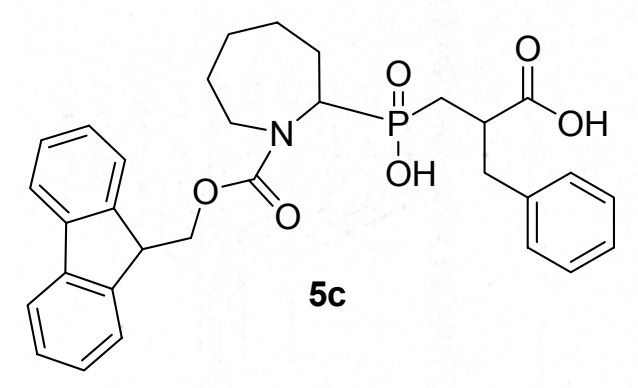

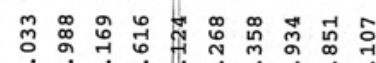

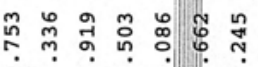

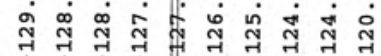

क 广

${ }^{13} \mathrm{C}$ NMR $\left(25^{\circ} \mathrm{C}, \mathrm{d}_{6}\right.$-DMSO $)$

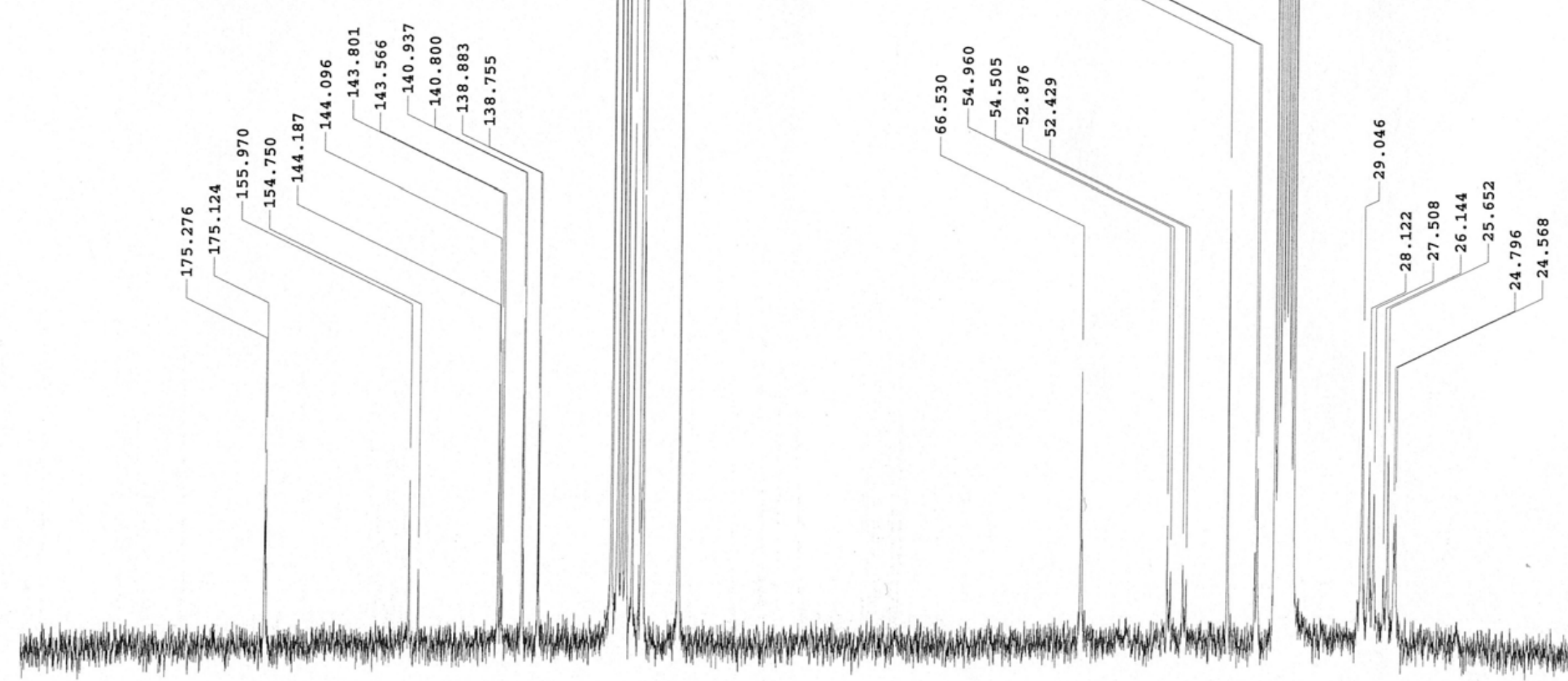

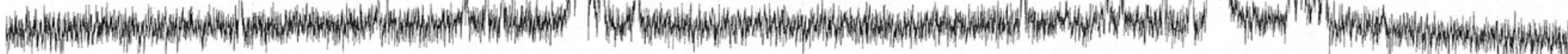

200

180

160

140

120

100

80

60

40

20

ppm 


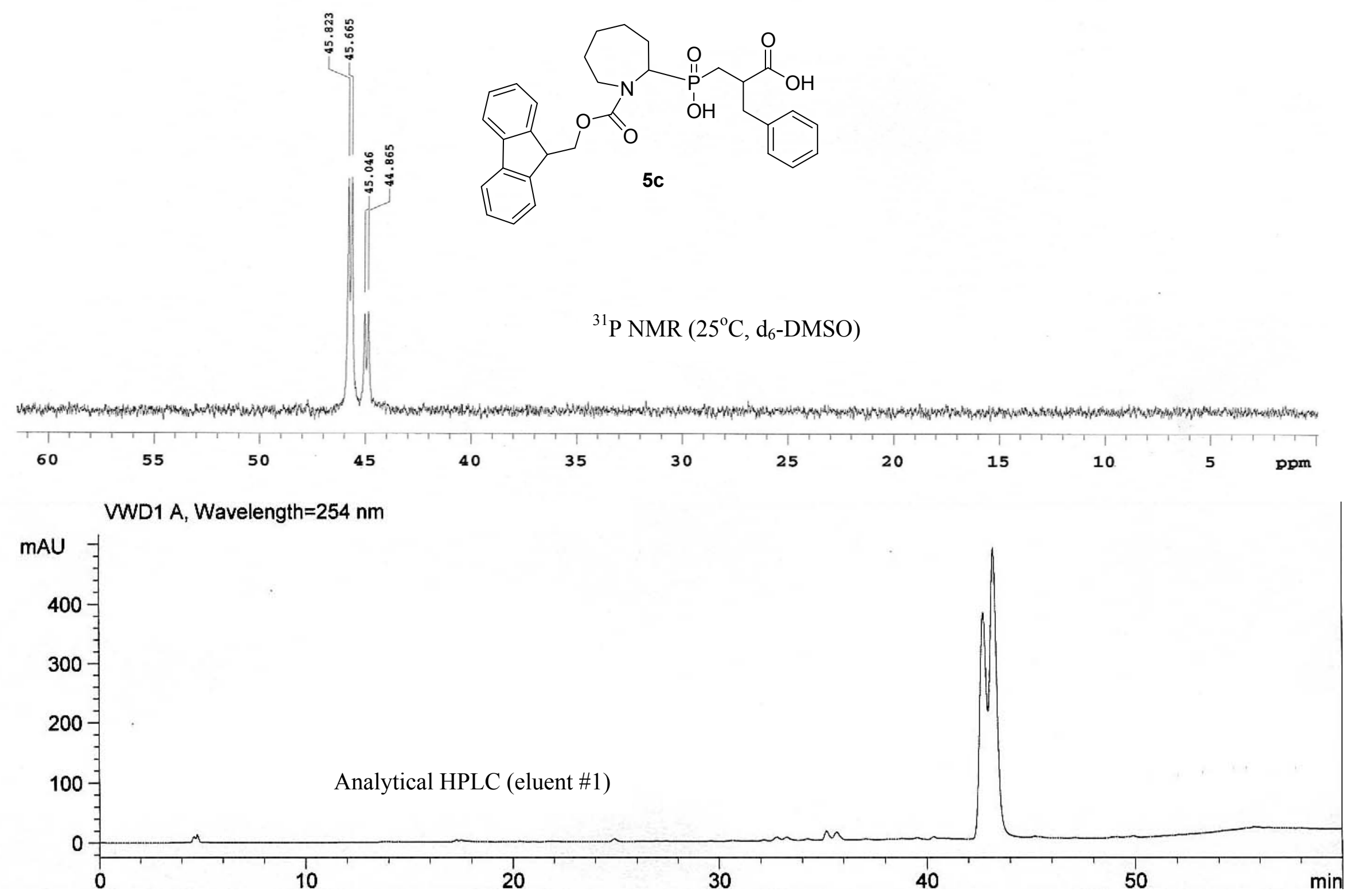



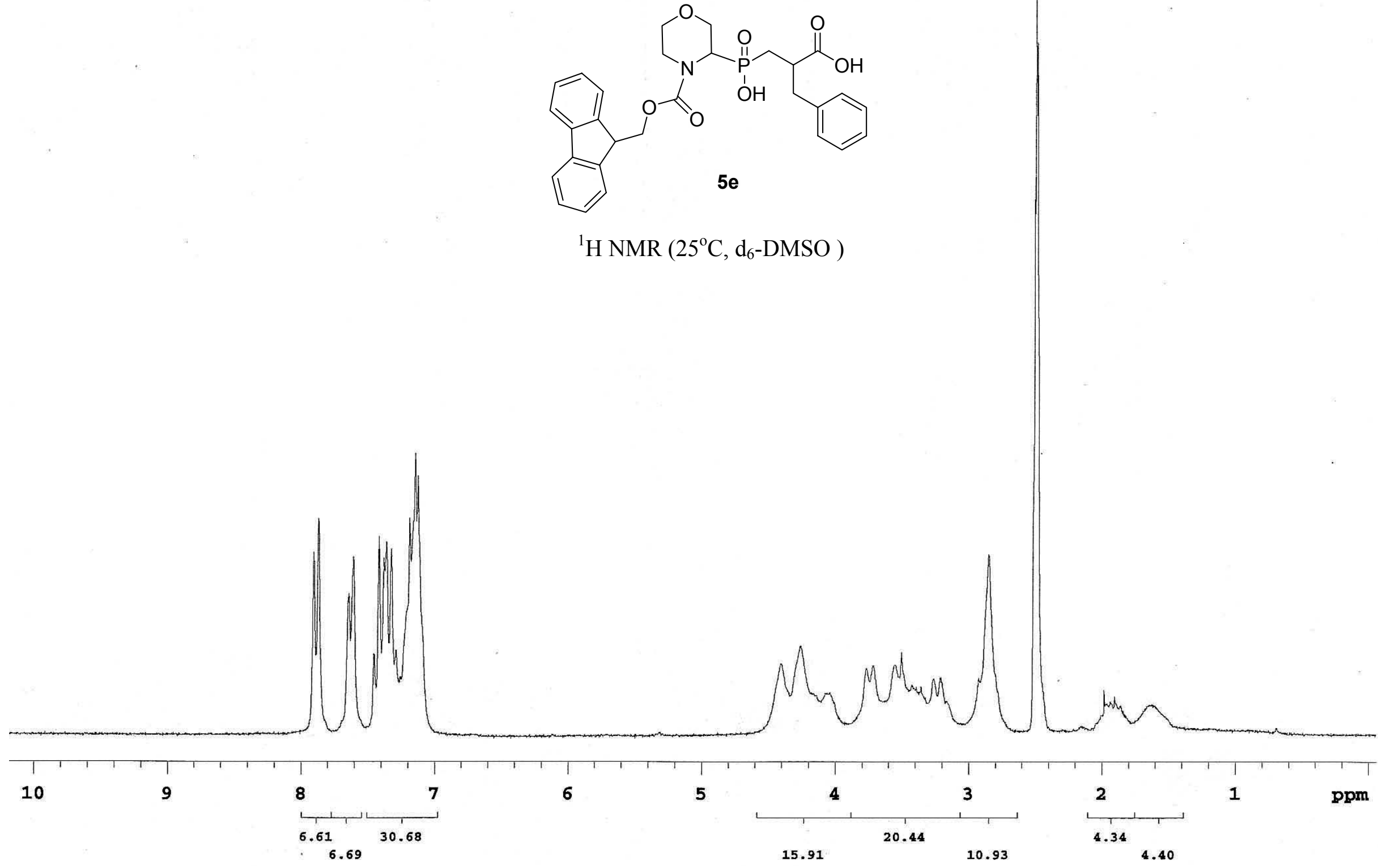


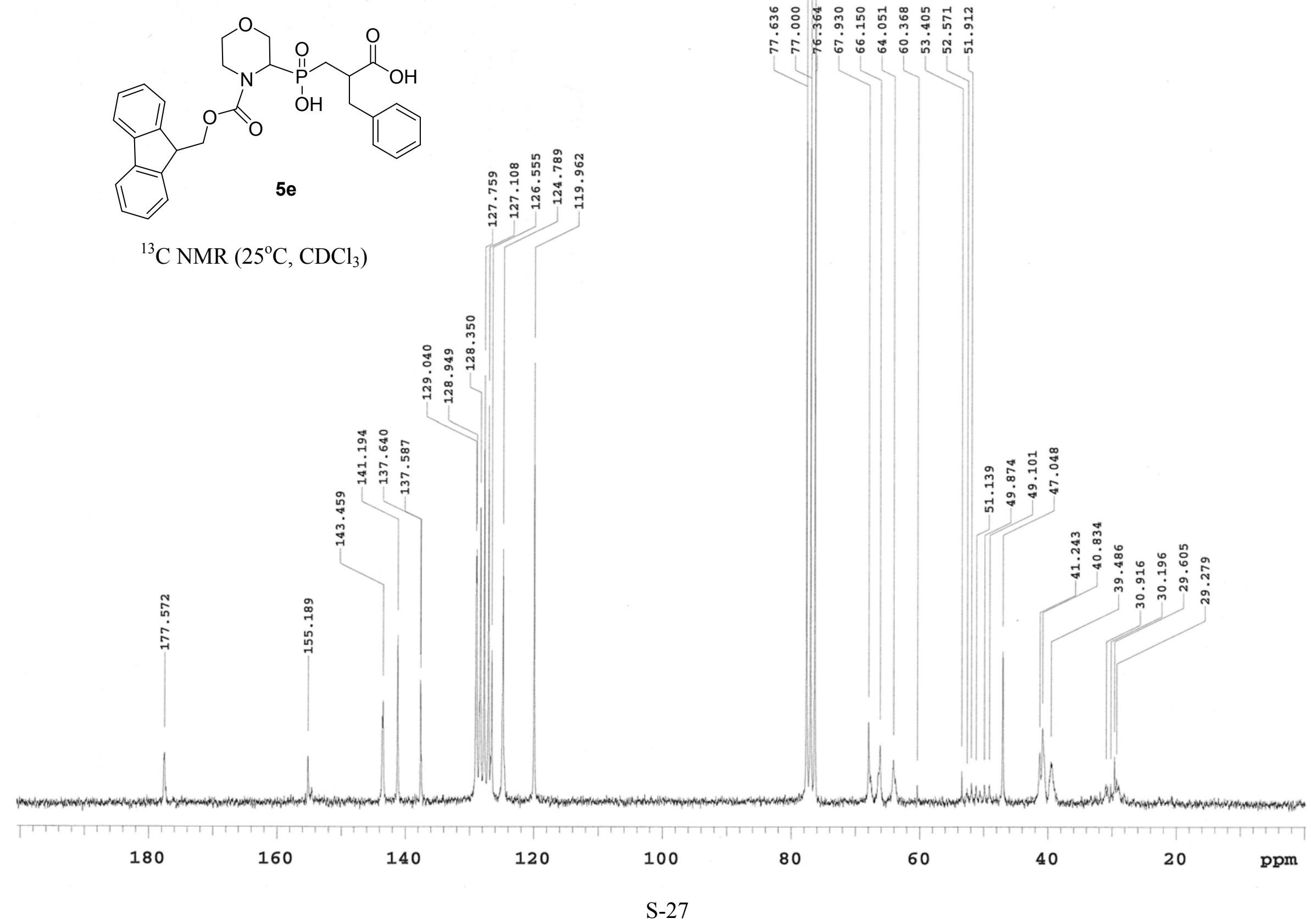



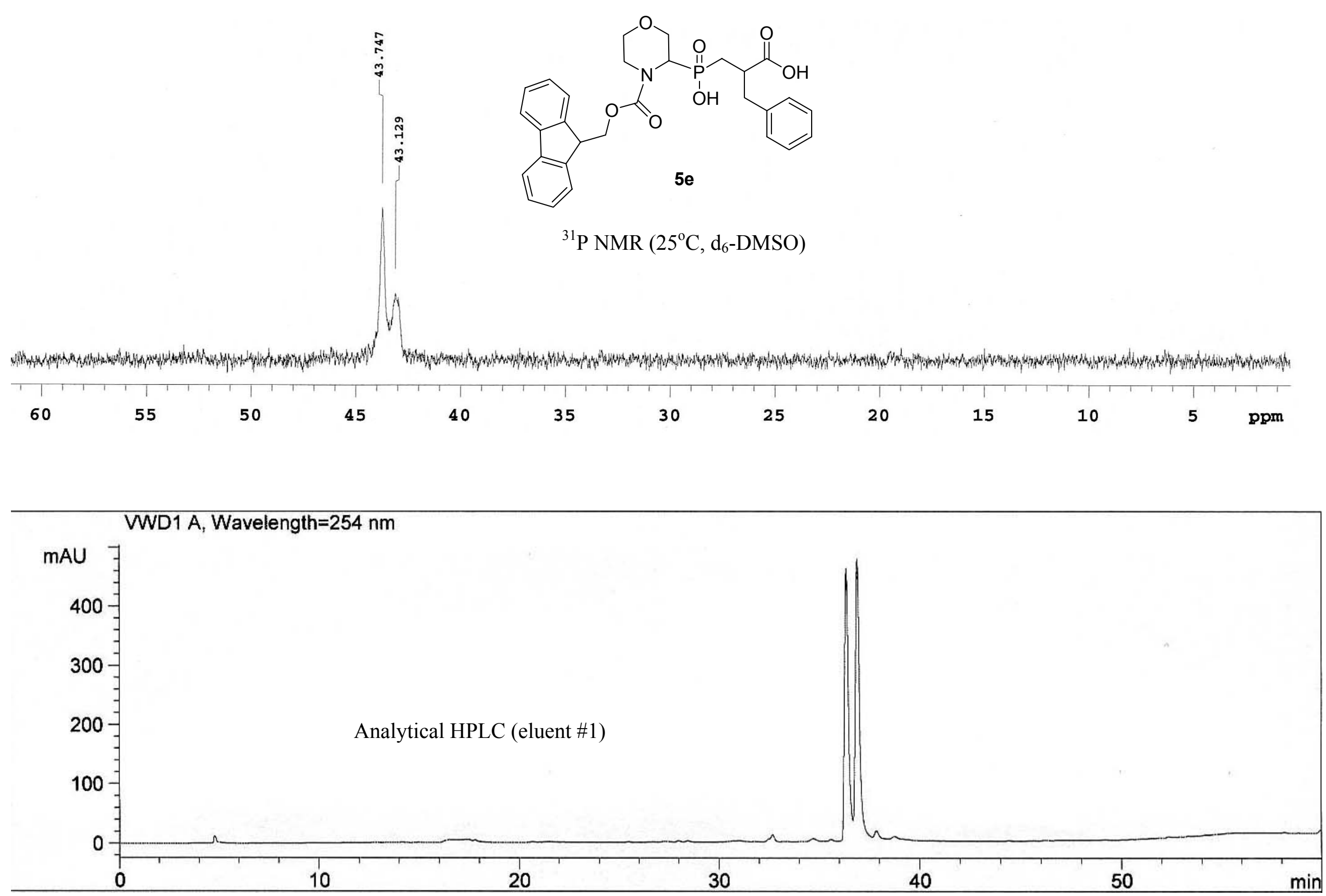